\title{
ON MONOMIAL IDEALS AND THEIR SOCLES
}

\author{
GEIR AGNARSSON AND NEIL EPSTEIN
}

\begin{abstract}
For a finite subset $M \subset\left[x_{1}, \ldots, x_{d}\right]$ of monomials, we describe how to constructively obtain a monomial ideal $I \subseteq R=K\left[x_{1}, \ldots, x_{d}\right]$ such that the set of monomials in $\operatorname{Soc}(I) \backslash I$ is precisely $M$, or such that $\bar{M} \subseteq R / I$ is a $K$-basis for the the socle of $R / I$. For a given $M$ we obtain a natural class of monomials $I$ with this property. This is done by using solely the lattice structure of the monoid $\left[x_{1}, \ldots, x_{d}\right]$. We then present some duality results by using anti-isomorphisms between upsets and downsets of $\left(\mathbb{Z}^{d}, \preceq\right)$. Finally, we define and analyze zero-dimensional monomial ideals of $R$ of type $k$, where type 1 are exactly the Artinian Gorenstein ideals, and describe the structure of such ideals that correspond to order-generic antichains in $\mathbb{Z}^{d}$.
\end{abstract}

\section{INTRODUCTION}

Aside from the algebraic benefits of the socle of a module over a commutative ring, when studying local rings, Cohen-Macaulay rings or Gorenstein rings [BH97, Eis95, Vil01] the socle of an ideal, in particular that of a monomial ideal of the polynomial ring over a field, has a rich lattice structure that many times can be studied solely by using combinatorial arguments. Consider the following: any range of mountains on a given piece of land where each mountain has the same cone-type shape is, of course, determined by the loci of the mountain tops. But is it determined by the mere valleys these mountains form? The answer depends on what additional conditions we know the mountain range has. This is one of the motivating questions in this article when we view a monomial ideal as a mountain range and the valleys it forms as the elements in the socle of the ideal that are not in the ideal.

For a field $K$, monomial ideals of the polynomial ring $R=K\left[x_{1}, \ldots, x_{d}\right]$ play a pivotal role in the investigation of Gröbner bases of general ideals of $R$ [BW93, AL94]. The underlying reason as to why monomial ideals are so convenient, yet so important, in investigating reduction systems for general ideals of $R$ stems in part from the fact that for every monomial ideal $I=\left(m_{1}, \ldots, m_{k}\right)$,

Date: February 1, 2018.

2010 Mathematics Subject Classification. 13B25, 13A02, 06A07.

Key words and phrases. poset, upset, downset, polynomial ring, monomial ideal, socle, duality, Artinian ideal, Gorenstein ideal, type $k$ monomial ideal. 
where no $m_{i}$ divides another $m_{j}$, has a Gröbner basis $M=\left\{m_{1}, \ldots, m_{k}\right\}$; exactly the minimum set of generators for $I$. For $A \subseteq\{1, \ldots, k\}$ let $m_{A}$ denote the least common multiple of the $m_{i}: i \in A$. With this convention one can define the Scarf complex $\Delta(I)$ of the monomial ideal $I$ as the simplicial complex consisting of all the subsets $A \subseteq\{1, \ldots, k\}$ with unique least common multiple $m_{A}$, that is

$$
\Delta(I)=\left\{A \subseteq\{1, \ldots, k\}: m_{A} \neq m_{B} \text { for all } B \neq A\right\} .
$$

The Scarf complex discussed in [BPS98] and [MS05] was first introduced in [Sca86]. It is easy to see that the facets $F_{d-1}(\Delta(I))$ of the Scarf complex $\Delta(I)$ are in bijective correspondence with the maximal monomials of $R \backslash I$ (w.r.t. the divisibility partial order), which is exactly the set of monomials of the socle $\operatorname{Soc}(I)$ that are not in $I$ (see definition in the following section.) The cardinality of this very set of monomials has many interesting combinatorial interpretations, two of which we will briefly describe here below.

We say that an ideal $I$ of $R$ is co-generated by a set $\mathcal{F}$ of $K$-linear functionals $R \rightarrow K$ if $I$ is the largest ideal of $R$ contained in all the kernels of the functionals in $\mathcal{F}$. A celebrated result by Macaulay from 1916 Mac94 states that every ideal of $R$ is finitely co-generated, so $R$ is coNoetherian in this sense. More specifically, it turns out that any monomial ideal $I$ of $R$ has at least $\left|F_{d-1}(\Delta(I))\right|$ co-generators and can always be co-generated by $\left|F_{d-1}(\Delta(I))\right|+1$ functionals (see Agn00). Further, for a given ideal $I$ (not necessarily monomial) of $R$ and a fixed term order, then $I$ has a Gröbner basis where the head or leading terms of the basis elements form a corresponding monomial ideal $L(I)$ of $R$, and $I$ can then be co-generated by one functional if $\left|F_{n-1}(\Delta(L(I)))\right|<d$ and by $\left|F_{n-1}(\Delta(L(I)))\right|+1$ functionals otherwise Agn00.

The cardinality $\left|F_{d-1}(\Delta(I))\right|$ is also linked to the number of edges in a simple graph on $k$ vertices in the following way. For given $d, k \in \mathbb{N}$ let $c_{d}(k)$ denote the maximum number of facets $\left|F_{d-1}(\Delta(I))\right|$ among all monomial ideals $I$ of $R$ that are minimally generated by $k$ monomials. In general, the Scarf complex $\Delta(I)$ is always a sub-complex of the boundary complex of a simplicial polytope $P(I)$ on $k$ vertices where one facet is missing. When $I$ is Artinian (or zero-dimensional) and generic (i.e. the powers of all $x_{i}$ in the generators for $I$ are distinct) then $\Delta(I)$ actually equals this boundary complex $P(I)$ with one facet missing [MS05. Using the Dehn-Sommerville equations for the simplicial polytope $P(I)$ one can show that determining $c_{d}(k)$ for $d \leq 5$ is equivalent to determining the maximum number of edges of the 1-skeleton of $P(I)$. This turns out to be the same problem of determining the maximum number of a simple graph on $k$ vertices of order dimension at most $d$ Agn97. 
We therefore see that the number of monomials in $\operatorname{Soc}(I) \backslash I$, the socle of the monomial ideal $I$ that are not in $I$, has some interesting combinatorial interpretations in addition to the known algebraic ones, in particular, the maximum number that the set $\operatorname{Soc}(I) \backslash I$ can have. Trivially we have $c_{2}(k)=k-1$ for any $k \in \mathbb{N}$ and less trivially we know that $c_{3}(k)=2 k-5$ for $k \geq 3$ Agn97, MS05. For each $d \geq 4$ the exact value of $c_{d}(k)$ is still unknown, although the asymptotic behavior does satisfy $c_{d}(k)=\Theta\left(k^{\lfloor d / 2\rfloor}\right)$ as $k$ tends to infinity and $d$ is fixed Agn97. In any case, we see from this that a monomial ideal minimally generated by $k$ monomials, can "generate" monomials in its socle of cardinality considerably larger order than that of $k$. In order for that to occur though, the $k$ monomials that generate the given ideal must be special and relate to each other in a singular way. By the same token, the resulting monomials in $\operatorname{Soc}(I) \backslash I$ also relate in a special way if they are generated by "few" generating monomials of $I$. Looking away from these extreme cases for a moment, some natural questions about monomial ideals and their socles, in particular about those monomials in the socle and not in the ideal, arise.

(i) Assume that for a given (apriori unknown) monomial ideal $I$ the set $\operatorname{Soc}(I) \backslash I$ is given, is it always possible to retrieve the ideal $I$ from it, if not, what additional structure is needed? In which cases is it unique?

(ii) Given any set of monomials that form an antichain w.r.t. divisibility order, can one always find a monomial ideal $I$ such that this set of monomials has the form $\operatorname{Soc}(I) \backslash I$ ? Is the ideal $I$ unique? If not, can they be characterized in some way?

The purpose of this article is to address these questions, discuss uniqueness and non-uniqueness, present up-down duality results, discuss generalizations to general ideals of $R$ and address what is similar with the monomial ideal case and what is not.

This article was in part inspired by the M.S. thesis of Anna-Rose Wolff [Wol16]. She analyzed the survival complex $\Sigma(R / I)$ of $R / I$ where $I$ is a monomial ideal that contains powers of all the variables of $R$. The survival complex is a simplicial complex whose vertices are the monomials of $R$ that are not in $I$, where a simplex consists of a set of monomials whose product is not in $I$. She showed [Wol16, Prop. 2.2.1] that the truly isolated points of that complex correspond to the monomial basis of the socle of $I$, a set which in this document we call $\partial \mathrm{oc}(I)$ (see Definition 2.1.) As such, it was natural for her to address the question we address here, completely solving the two-variable case [Wol16, Algorithm 3.3.1 and Proposition 3.3.3] and making headway on the three-variable case [Wol16, Algorithm 3.4.1].

The rest of this article is organized as follows: 
In Section 2 we define some basic concepts about partially ordered sets that we will be using and referring to throughout the article.

In Section 3 we show that if for a monomial ideal $I$ the set $\operatorname{Soc}(I) \backslash I$ is given, then we can retrieve a monomial ideal $I$ back from a derived version of the set $\operatorname{Soc}(I) \backslash I$.

In Section 4 we present and use an intuitively obvious duality between monomial ideals of $R$ on the one hand and "down-sets" of monomials on the other hand, that contain all elements dividing a given element of the down-set (see definitions in Section 4).

In Section 5 we elaborate more explicitly the discussion presented in Section 3 and show that if $\operatorname{Soc}(I) \backslash I$ is given, then there is a unique zero-dimensional ideal $I$ yielding the given set of monomials. As a corollary we obtain a well-known description of Artinian monomial ideals of $R$ that are Gorenstein. We then use what we have established to describe Artinian monomial ideals that are type 2 Gorenstein (where type 1 Gorenstein is the usual Gorenstein notion (see definitions in Section (5)).

In Section 6 we discuss further Artinian type $k$ Gorenstein monomial ideals and describe their order-generic case.

In Section 7 we discuss the socle of ideals of $R=K\left[x_{1}, \ldots, x_{d}\right]$ in more general settings when $K$ is an algebraically closed field.

Finally, in Section 8 we summarize our main results and pose some questions.

\section{Partially ordered Sets, Basic Definitions And notations}

The set of integers will be denoted by $\mathbb{Z}$, the set of natural numbers by $\mathbb{N}$ and the set of nonnegative numbers $\mathbb{N} \cup\{0\}$ by $\mathbb{N}_{0}$. For each $n \in \mathbb{N}$ we let $[n]:=\{1, \ldots, n\}$.

Throughout, $R=K\left[x_{1}, \ldots, x_{d}\right]$ will denote the polynomial ring over a field $K$. By the socle of an ideal $I \subseteq R$ w.r.t. the maximal ideal $\mathbf{m}=\left(x_{1}, \ldots, x_{d}\right)$ of $R$, we will mean the ideal

$$
\operatorname{Soc}(I)=\operatorname{Soc}_{\mathbf{m}}(I):=(I: \mathbf{m})=\left\{f \in R: x_{i} f \in I \text { for each } i\right\} .
$$

Note that for a monomial ideal $I \subseteq R$, the set $\operatorname{Soc}(I) \backslash I$ contains monomials $a$ such that (i) $a \notin I$ and (ii) $x_{i} a \in I$ for every $i \in[d]$.

Definition 2.1. For a monomial ideal $I$ and the maximal ideal $\mathbf{m}=\left(x_{1}, \ldots, x_{d}\right)$ of $R$, let $\partial \operatorname{oc}(I)$ denote the set of the monimials in $\operatorname{Soc}(I) \backslash I$. 


\section{Remarks.}

(i) By the above Definition 2.1, we have $\operatorname{Soc}(I)=I \oplus \operatorname{Span}_{K}(\partial \mathrm{oc}(I))$ as a $K$-vector spaces.

(ii) The image $\overline{\operatorname{Soc}(I)}=(\overline{0}: \mathbf{m} / I)=\operatorname{Soc}(R / I)$ in $R / I$ has a basis as a vector space over $K$ consisting of the images of the monomials in $\partial \mathrm{oc}(I)$ (see [Vil01, AF74.) Hence, $\partial \mathrm{oc}(I)$ consists exactly of the maximal monomials in $R \backslash I$ w.r.t. the divisibility partial order and $|\partial \mathrm{oc}(I)|=\operatorname{dim}_{K}(\operatorname{Soc}(R / I))$.

Assume for a moment we have a general ideal $I$ and a general maximal ideal $\mathbf{m}$ of $R$. If $\mathbf{m}$ does not contain $I$, then $\mathbf{m}+I=R$ and hence for $f \in(I: \mathbf{m})$ we have $f \in f I+f \mathbf{m} \subseteq I$. Therefore $(I: \mathbf{m})=I$ and so $\operatorname{Soc}_{\mathbf{m}}(I)$ is trivial. Therefore, for an ideal $I$ and a maximal ideal $\mathbf{m}$ of $R$, the socle $\operatorname{Soc}_{\mathbf{m}}(I)$ is only of interest when $I \subseteq \mathbf{m}$. Hence, unless otherwise stated, we will always assume $I \subseteq \mathbf{m}$.

Most of what we establish from now on about monomial ideals of $R=K\left[x_{1}, \ldots, x_{d}\right]$ will only use the the monoid $\left[x_{1}, \ldots, x_{d}\right]$ as a partially ordered set (poset) with the partial order given by divisibility. We present some basic definitions and notations for general posets that we will use throughout the article.

For a poset $(P, \leq)$ recall that $C \subseteq P$ is a chain if $C$ forms a linearly or totally ordered set within $(P, \leq)$. A subset $N \subseteq P$ is an antichain or a clutter if no two elements in $N$ are comparable in $(P, \leq)$.

For a poset $(P, \leq)$ call a subset $U \subseteq P$ an upset, or an up-filter, of $P$ if $x \geq u \in U \Rightarrow x \in U$. Call a subset $D \subseteq P$ a downset, or a down-filter, of $P$ if if $x \leq d \in D \Rightarrow x \in D$.

For an upset $U$ of a poset $P$ let $S_{d}(U):=\max (P \backslash U)$ be the maximal elements of $P \backslash U$. For a downset $D$ of a poset $P$ let $S_{u}(D):=\min (P \backslash D)$ be the minimal elements of $P \backslash D$.

For a subset $A \subseteq P$, let $U(A):=\{x \in P: x \geq a$ for some $a \in A\}$ be the upset generated by $A$, and $D(A):=\{x \in P: x \leq a$ for some $a \in A\}$ the downset generated by $A$. If $A=\left\{a_{1}, \ldots, a_{n}\right\}$ is finite, then we write $U\left(a_{1}, \ldots, a_{n}\right)$ (resp. $\left.D\left(a_{1}, \ldots, a_{n}\right)\right)$ for $U(A)(\operatorname{resp} . D(A)$.)

Recall that $\mathbb{Z}^{d}$ has a natural partial order $\preceq$ where for $\tilde{a}=\left(a_{1}, \ldots, a_{d}\right)$ and $\tilde{b}=\left(b_{1}, \ldots, b_{d}\right)$ we have $\tilde{a} \preceq \tilde{b} \Leftrightarrow a_{i} \leq b_{i}$ for each $i \in[d]$. This is a generalization of the partial order given in [GP06, Ex. 19, p. 71], and we will henceforth be using " $\leq$ " for this partial order " $\preceq$ ". The usual componentwise addition + , which makes $\left(\mathbb{Z}^{d},+\right)$ an abelian group, respects the mention partial order $\leq$ 
For any fixed point $\tilde{c} \in \mathbb{Z}^{d}$, the map $\tau_{\tilde{c}}: \mathbb{Z}^{d} \rightarrow \mathbb{Z}^{d}$ given by $\tau_{\tilde{c}}(\tilde{x})=\tilde{c}+\tilde{x}$ is an order preserving translation, and hence an order automorphism, of the poset $\left(\mathbb{Z}^{d}, \leq\right)$. The following is easy to obtain.

Observation 2.2. For any $A \subseteq \mathbb{Z}^{d}$ and $\tilde{c} \in \mathbb{Z}^{d}$ we have

$$
\tau_{\tilde{c}}(U(A))=U\left(\tau_{\tilde{c}}(A)\right), \quad \tau_{\tilde{c}}(D(A))=D\left(\tau_{\tilde{c}}(A)\right) .
$$

Further, for any upset $U \subseteq \mathbb{Z}^{d}$ and downset $D \subseteq \mathbb{Z}^{d}$ we have

$$
\tau_{\tilde{c}}\left(S_{d}(U)\right)=S_{d}\left(\tau_{\tilde{c}}(U)\right), \quad \tau_{\tilde{c}}\left(S_{u}(D)\right)=S_{u}\left(\tau_{\tilde{c}}(D)\right)
$$

Writing out what the above observation states in terms of $\tilde{c}$ we get

$$
\begin{array}{cc}
U(A+\tilde{c})=U(A)+\tilde{c}, \quad D(A+\tilde{c})=D(A)+\tilde{c}, \\
S_{d}(U+\tilde{c})=S_{d}(U)+\tilde{c}, \quad S_{u}(D+\tilde{c})=S_{u}(D)+\tilde{c} .
\end{array}
$$

An upset $U \subseteq \mathbb{Z}^{d}$ is cofinite if here is a $\tilde{c} \in \mathbb{Z}^{d}$ such that (i) $U \subseteq U(\tilde{c})$ and (ii) $U(\tilde{c}) \backslash U$ is finite. Likewise a downset $D \subseteq \mathbb{Z}^{d}$ is cofinite if there is a $\tilde{c} \in \mathbb{Z}^{d}$ such that (i) $D \subseteq D(\tilde{c})$ and (ii) $D(\tilde{c}) \backslash D$ is finite.

The map given by $\tilde{p}=\left(p_{1}, \ldots, p_{d}\right) \mapsto x_{1}^{p_{1}} \cdots x_{d}^{p_{d}}=\tilde{x}^{\tilde{p}}$ is an isomorphism between the additive monoid $\mathbb{N}_{0}^{d}$ and the multiplicative monoid $\left[x_{1}, \ldots, x_{d}\right]$. Moreover, the map is also an order isomorphism from the poset $\left(\mathbb{N}_{0}^{d}, \leq\right)$, where $\leq$ is the partial order of $\mathbb{Z}^{d}$ from above, to $\left[x_{1}, \ldots, x_{d}\right]$ as a poset given by divisibility. Hence as the map is an order preserving monoid isomorphism, we will for the most part in the next two sections deal with the additive monoid $\mathbb{N}_{0}^{d}$ and the abelian group $\mathbb{Z}^{d}$ instead of the multiplicative monoid $\left[x_{1}, \ldots, x_{d}\right]$ within the ring $R$. Via this isomorphism we have a bijective correspondence between monomial ideals of $R$ and upsets of the poset $\mathbb{N}_{0}^{d}$.

\section{Retrieving AN UPSET FROM MAXimal ElEMENTS NOT IN THE UPSET}

We will in this section and the following section describe everything in terms of $\mathbb{N}_{0}^{d}$ or $\mathbb{Z}^{d}$ instead of the ring $R=K\left[x_{1}, \ldots, x_{d}\right]$ as we justified in the last paragraph in the previous section.

Let $G=\left\{\tilde{p}_{1}, \ldots, \tilde{p}_{k}\right\} \subseteq \mathbb{N}^{d}$ be an antichain. For the moment we assume that $G$ is positive, i.e. all the coordinates of each $\tilde{p}_{i}$ are strictly positive. (This is by no means a restrictive assumption as will become clear as we go along.) If $\tilde{p}_{i}=\left(p_{i 1}, \ldots, p_{i d}\right)$ for each $i$, then for each $j \in[d]$ let

$$
m_{j}=\min \left\{p_{i j}: 1 \leq i \leq d\right\}-1, \quad M_{j}=\max \left\{p_{i j}: 1 \leq i \leq d\right\}+1
$$


and

$$
\begin{aligned}
& B^{*}(G)= \\
& \quad\left\{\left(M_{1}, m_{2}, m_{3} \ldots, m_{d}\right),\left(m_{1}, M_{2}, m_{3}, \ldots, m_{d}\right),\left(m_{1}, m_{2}, M_{3}, \ldots, m_{d}\right),\right. \\
& \left.\quad \ldots,\left(m_{1}, m_{2}, m_{3}, \ldots, M_{d}\right)\right\} .
\end{aligned}
$$

Let $G^{*}=G \cup B^{*}(G)$. That $\tilde{r} \in S_{d}(U(G))$, means precisely that $\tilde{r} \notin U(G)$ and $\tilde{r}+\tilde{e}_{j} \in U(G)$ for each $j$, where $\tilde{e}_{j}$ is the usual basis vector for $\mathbb{R}^{d}$ with 1 in the $j$-coordinate and 0 everywhere else. Since $\tilde{r} \notin U(G)$ and $\tilde{r}+\tilde{e}_{j} \in U(G)$ for each given $j$, then for some $\tilde{p}_{i} \in G$ we have $r_{j}=p_{i j}-1<M_{j}$. Since this holds for each $j$, then $\tilde{r}$ cannot be greater than any element of $B^{*}(G)$ and so $\tilde{r} \notin U\left(B^{*}(G)\right)$ and hence $\tilde{r} \notin U\left(G^{*}\right)$. Since $G \subseteq G^{*}$ and so $U(G) \subseteq U\left(G^{*}\right)$, we have $\tilde{r}+\tilde{e}_{j} \in U\left(G^{*}\right)$ for each $j$, and so $\tilde{r} \in S_{d}\left(U\left(G^{*}\right)\right)$.

Also we have $U(G) \subseteq U\left(G^{*}\right) \subseteq U(\tilde{m})$, where $\tilde{m}=\left(m_{1}, \ldots, m_{d}\right)$ from (10), and $U(\tilde{m}) \backslash U\left(G^{*}\right) \subseteq$ $U(\tilde{m}) \backslash U\left(B^{*}(G)\right)$, which is finite. We summarize in the following.

Proposition 3.1. For any antichain $G=\left\{\tilde{p}_{1}, \ldots, \tilde{p}_{k}\right\} \subseteq \mathbb{N}^{d}$ we have

$$
S_{d}(U(G)) \subseteq S_{d}\left(U\left(G^{*}\right)\right) \subseteq U(\tilde{m}) \backslash U\left(G^{*}\right),
$$

which is a finite set, and hence $U\left(G^{*}\right)$ is cofinite.

Note that the $S_{d}\left(U\left(G^{*}\right)\right)$ corresponds to the socle of the monomial ideal whose generators correspond to $G^{*}$. The main result in this section is the following.

Theorem 3.2. For a given antichain $G=\left\{\tilde{p}_{1}, \ldots, \tilde{p}_{k}\right\} \subseteq \mathbb{N}^{d}$ we have

$$
G=S_{u}\left(D\left(S_{d}\left(U\left(G^{*}\right)\right)\right)\right) .
$$

Before proving Theorem 3.2 we need the following.

Lemma 3.3. Let $(P, \leq)$ be a poset. For an upset $U \subseteq P$ and a downset $D \subseteq P$ we have

$$
D\left(S_{d}(U)\right) \cap U=U\left(S_{u}(D)\right) \cap D=\emptyset .
$$

Proof. If $\tilde{x} \in D\left(S_{u}(U)\right)$, then $\tilde{x} \leq \tilde{y} \in S_{d}(U)$. If now $\tilde{x} \in U$ as well, then by definition of an upset we must have $\tilde{y} \in U$, which is a contradiction since $U \cap S_{d}(U)=\emptyset$. In the same way we obtain $U\left(S_{u}(D)\right) \cap D=\emptyset$. 


\section{Conventions.}

(i) For a set $A \subseteq \mathbb{Z}^{d}$ and $i \in[d]$ let $A_{x_{i}=a_{i}}:=\left\{\tilde{x} \in A: x_{i}=a_{i}\right\}$.

(ii) For a finite set $G \subseteq \mathbb{Z}^{d}$ let $\bar{U}(G)=U(\tilde{m}) \backslash U\left(G^{*}\right)$, where $\tilde{m}$ is as in (1), which then is a finite set.

(iii) For $\mathcal{I}=\left\{i_{1}, \ldots, i_{h}\right\} \subseteq[d]$ let $\pi_{\mathcal{I}}=\pi_{i_{1}, \ldots, i_{h}}: \mathbb{R}^{d} \rightarrow \mathbb{R}^{h}$ denote the natural projection onto coordinates $i_{1}, \ldots, i_{h}$. In particular $\pi_{i}(\tilde{x})=x_{i}$ and $\pi_{\hat{\imath}}(\tilde{x})=\left(x_{1}, \ldots, x_{i-1}, x_{i+1}, \ldots, x_{d}\right)$.

Proof of Theorem [3.2. Let $\tilde{p}=\left(p_{1}, \ldots, p_{d}\right) \in G$. Since $U\left(G^{*}\right)$ is cofinite, then for each $i \in[d]$ the set $\bar{U}(G)_{x_{i}=p_{i}-1}$ is also a finite poset. Also, it is nonempty since $\tilde{p}-\tilde{e}_{i}=\left(p_{1}, \ldots, p_{i-1}, p_{i}-\right.$ $\left.1, p_{i+1}, \ldots, p_{d}\right) \in \bar{U}(G)_{x_{i}=p_{i}-1}$ as $G^{*}$ is an antichain. Hence, there is a maximal element $\tilde{q} \in$ $\bar{U}(G)_{x_{i}=p_{i}-1}$ with $\tilde{p}-\tilde{e}_{i} \leq \tilde{q}$. Since $\tilde{q}+\tilde{e}_{i} \geq \tilde{p}$, then $\tilde{q}+\tilde{e}_{i} \in U(\tilde{p}) \subseteq U(G) \subseteq U\left(G^{*}\right)$. Also, since $\tilde{q}$ is maximal in $\bar{U}(G)_{x_{i}=p_{i}-1}$, we have $\tilde{q}+\tilde{e}_{\ell} \in U\left(G^{*}\right)_{x_{i}=p_{i}-1} \subseteq U\left(G^{*}\right)$ for all $\ell \neq i$, and so $\tilde{q} \in S_{d}\left(U\left(G^{*}\right)\right)$. So, for each $i \in[d]$ we have $\tilde{p}-\tilde{e}_{i} \in D\left(S_{d}\left(U\left(G^{*}\right)\right)\right)$. Since $\tilde{p} \in G \subseteq U\left(G^{*}\right)$, then by Lemma 3.3 we have $\tilde{p} \notin D\left(S_{d}\left(U\left(G^{*}\right)\right)\right)$, and therefore $\tilde{p} \in S_{u}\left(D\left(S_{d}\left(U\left(G^{*}\right)\right)\right)\right)$. This proves that $G \subseteq S_{u}\left(D\left(S_{d}\left(U\left(G^{*}\right)\right)\right)\right)$.

For the other direction, we first verify that

$$
S_{u}\left(D\left(S_{d}\left(U\left(G^{*}\right)\right)\right)\right) \subseteq U(\tilde{m}+\tilde{1}),
$$

where $\tilde{1}=(1, \ldots, 1) \in \mathbb{Z}^{d}$. First note that by Proposition 3.1 we have $S_{d}\left(U\left(G^{*}\right)\right) \subseteq U(\tilde{m}) \backslash U\left(G^{*}\right) \subseteq$ $U(\tilde{m})$.

If $\tilde{r} \in S_{u}\left(D\left(S_{d}\left(U\left(G^{*}\right)\right)\right)\right)$, then $\tilde{r}-\tilde{e}_{i} \in D\left(S_{d}\left(U\left(G^{*}\right)\right)\right)$ for each $i$, and so $\tilde{r}-\tilde{e}_{i} \leq \tilde{q}$ for some $\tilde{q} \in S_{d}\left(U\left(G^{*}\right)\right) \subseteq U(\tilde{m})$ for each $i$. Consider a fixed $i$. Since $\tilde{r} \not \leq \tilde{q}$ and $\tilde{r}-\tilde{e}_{i} \leq \tilde{q}$, we must have $r_{i}-1=q_{i}$. Since $\tilde{q} \in S_{d}\left(U\left(G^{*}\right)\right) \subseteq U(\tilde{m})$, we have $q_{i} \geq m_{i}$ and therefore $r_{i}=q_{i}+1 \geq m_{i}+1$. Since this holds for each $i$, we have thus (3).

For $\tilde{r} \in S_{u}\left(D\left(S_{d}\left(U\left(G^{*}\right)\right)\right)\right)$ we have for each $i$ that $\tilde{r}-\tilde{e}_{i} \in D\left(S_{d}\left(U\left(G^{*}\right)\right)\right)$, and hence, by Lemma 3.3, $\tilde{r}-\tilde{e}_{i} \notin U\left(G^{*}\right)$ for each $i$. If now $\tilde{r} \in U\left(G^{*}\right)$, then $\tilde{r}$ is a minimal element of $U\left(G^{*}\right)$ and hence $\tilde{r} \in G^{*}$. Hence

$$
\tilde{r} \in G^{*} \cap S_{u}\left(D\left(S_{d}\left(U\left(G^{*}\right)\right)\right)\right) \subseteq G^{*} \cap U(\tilde{m}+\tilde{1})=G .
$$

Therefore it suffices to show that $S_{u}\left(D\left(S_{d}\left(U\left(G^{*}\right)\right)\right)\right) \subseteq U\left(G^{*}\right)$.

Assume $\tilde{r} \in S_{u}\left(D\left(S_{d}\left(U\left(G^{*}\right)\right)\right)\right) \backslash U\left(G^{*}\right)$. We then have $\tilde{r} \in U(\tilde{m}+\tilde{1}) \backslash U\left(G^{*}\right) \subseteq U(\tilde{m}) \backslash U\left(G^{*}\right)$. By Proposition $3.1 U\left(G^{*}\right)$ is cofinite, and so there is a maximal element $\tilde{q} \in U(\tilde{m}) \backslash U\left(G^{*}\right)$ with $\tilde{r} \leq \tilde{q}$. In this case we have, by the definition of $S_{d}$, that $\tilde{r} \leq \tilde{q} \in S_{d}\left(U\left(G^{*}\right)\right)$ and hence $\tilde{r} \in D\left(S_{d}\left(U\left(G^{*}\right)\right)\right)$ 
contradicting that $\tilde{r} \in S_{u}\left(D\left(S_{d}\left(U\left(G^{*}\right)\right)\right)\right)$. This proves that $S_{u}\left(D\left(S_{d}\left(U\left(G^{*}\right)\right)\right)\right) \subseteq U\left(G^{*}\right)$, and hence, by previous paragraph, $S_{u}\left(D\left(S_{d}\left(U\left(G^{*}\right)\right)\right)\right) \subseteq G$.

Remark. The values $m_{1}, \ldots, m_{d}$ and $M_{1}, \ldots, M_{d}$ from (11), used to define $G^{*}$, do not play a major role, except for merely being small and respectively large enough. In fact, if $\tilde{a} \leq \tilde{m}$ and $\tilde{b} \geq \tilde{M}$ and

$$
B^{*}(\tilde{a}, \tilde{b})=\left\{\left(b_{1}, a_{2}, a_{3} \ldots, a_{d}\right),\left(a_{1}, b_{2}, a_{3}, \ldots, a_{d}\right),\left(a_{1}, a_{2}, b_{3}, \ldots, a_{d}\right), \ldots,\left(a_{1}, a_{2}, a_{3}, \ldots, b_{d}\right)\right\}
$$

then we can define $G^{*}(\tilde{a}, \tilde{b})=G \cup B^{*}(\tilde{a}, \tilde{b})$, and we then, as in Proposition 3.1, obtain that

$$
S_{d}(U(G)) \subseteq S_{d}\left(U\left(G^{*}(\tilde{a}, \tilde{b})\right)\right) \subseteq U(\tilde{a}) \backslash U\left(G^{*}(\tilde{a}, \tilde{b})\right)
$$

which is a finite set, and hence $U\left(G^{*}(\tilde{a}, \tilde{b})\right)$ is cofinite, and the proof of Theorem 3.2 works verbatim for the following slight generalization.

Theorem 3.4. For a given antichain $G=\left\{\tilde{p}_{1}, \ldots, \tilde{p}_{k}\right\} \subseteq \mathbb{N}^{d}$ and for $\tilde{a} \leq \tilde{m}$ and $\tilde{b} \geq \tilde{M}$ where $\tilde{m}$ and $\tilde{M}$ are as in (1), we have

$$
G=S_{u}\left(D\left(S_{d}\left(U\left(G^{*}(\tilde{a}, \tilde{b})\right)\right)\right)\right)
$$

This will be used in the next Section 4 .

Note that Theorem 3.2 states that one can retrieve the antichain, or the generating points, $G$ from $S_{d}\left(U\left(G^{*}\right)\right)$ alone. However, this does not mean that $S_{d}\left(U\left(G^{*}\right)\right)$ can be arbitrary; the set is derived from the (apriori unknown) set $G$.

In the next section we use poset duality of $\mathbb{Z}^{d}$ to obtain some related results from Theorem 3.2 where we start with an arbitrary antichain $Q$ and show how it corresponds to the socle of a certain monomial ideal.

\section{UP-DOWN DUALITY}

As in the derivation of Observation 2.2 , for any fixed point $\tilde{c} \in \mathbb{Z}^{d}$, the map $\rho_{\tilde{c}}: \mathbb{Z}^{d} \rightarrow \mathbb{Z}^{d}$ given by $\rho_{\tilde{c}}(\tilde{x})=\tilde{c}-\tilde{x}$ is a reverse-order preserving rotation, and hence an anti-automorphism of the poset $\left(\mathbb{Z}^{d}, \leq\right)$. In particular, for $N \subseteq \mathbb{Z}^{d}$ we have that $N$ is an antichain iff $\rho_{\tilde{c}}(N)$ is an antichain. Clearly $\rho_{\tilde{c}}$ is its own inverse, and so we have the following.

Observation 4.1. For any $\tilde{c} \in \mathbb{Z}^{d}$ we have $\rho_{\tilde{c}}^{2}=I_{\mathbb{Z}^{d}}$, the identify map of $\mathbb{Z}^{d}$. For any $A \subseteq \mathbb{Z}^{d}$ and $\tilde{c} \in \mathbb{Z}^{d}$ we have

$$
\rho_{\tilde{c}}(U(A))=D\left(\rho_{\tilde{c}}(A)\right), \quad \rho_{\tilde{c}}(D(A))=U\left(\rho_{\tilde{c}}(A)\right)
$$


Further, for any upset $U \subseteq \mathbb{Z}^{d}$ and downset $D \subseteq \mathbb{Z}^{d}$ then $\rho_{\tilde{c}}(U)$ is a downset, $\rho_{\tilde{c}}(D)$ is an upset and

$$
\rho_{\tilde{c}}\left(S_{d}(U)\right)=S_{u}\left(\rho_{\tilde{c}}(U)\right), \quad \rho_{\tilde{c}}\left(S_{u}(D)\right)=S_{d}\left(\rho_{\tilde{c}}(D)\right) .
$$

Let $G=\left\{\tilde{p}_{1}, \ldots, \tilde{p}_{k}\right\} \subseteq \mathbb{N}^{d}$ be an antichain, and $G^{*}=G \cup B^{*}(G)$, where $B^{*}(G)$ is as in (2). If $\rho=\rho_{\tilde{m}+\tilde{M}}$ where $\tilde{m}$ and $\tilde{M}$ are as in (1), then for any coordinate $i$ we have

$\min \left(\pi_{i}(\rho(G))\right)=m_{i}+M_{i}-\left(M_{i}-1\right)=m_{i}+1, \quad \max \left(\pi_{i}(\rho(G))\right)=m_{i}+M_{i}-\left(m_{i}+1\right)=M_{i}-1$, and so $G, \rho(G) \subseteq U(\tilde{m}+\tilde{1}) \cap D(\tilde{M}-\tilde{1})$.

Suppose that $Q \subseteq \mathbb{N}^{d}$ is such that $\min \left(\pi_{i}(Q)\right)=m_{i}+1$ and $\max \left(\pi_{i}(Q)\right)=M_{i}-1$ for each $i$. Since $\rho$ is its own inverse, there is a unique $G \subseteq U(\tilde{m}+\tilde{1}) \cap D(\tilde{M}-\tilde{1}$ ) with $Q=\rho(G)$ (namely $G=\rho(Q)$,) such that $\min \left(\pi_{i}(G)\right)=m_{i}+1$ and $\max \left(\pi_{i}(G)\right)=M_{i}-1$ for each $i$, as well. Hence we have

$$
\begin{aligned}
\rho\left(G^{*}\right)= & \rho\left(G \cup B^{*}(G)\right) \\
= & \rho(G) \cup \rho\left(B^{*}(G)\right) \\
= & Q \cup\left\{\left(m_{1}, M_{2}, M_{3} \ldots, M_{d}\right),\left(M_{1}, m_{2}, M_{3}, \ldots, M_{d}\right),\left(M_{1}, M_{2}, m_{3}, \ldots, M_{d}\right),\right. \\
& \left.\ldots,\left(M_{1}, M_{2}, M_{3}, \ldots, m_{d}\right)\right\} .
\end{aligned}
$$

Hence, if we define

$$
\begin{aligned}
& B_{*}(Q)= \\
& \quad\left\{\left(m_{1}, M_{2}, M_{3} \ldots, M_{d}\right),\left(M_{1}, m_{2}, M_{3}, \ldots, M_{d}\right),\left(M_{1}, M_{2}, m_{3}, \ldots, M_{d}\right),\right. \\
& \left.\quad \ldots,\left(M_{1}, M_{2}, M_{3}, \ldots, m_{d}\right)\right\},
\end{aligned}
$$

where for each $i \min \left(\pi_{i}(Q)\right)=m_{i}+1$ and $\max \left(\pi_{i}(Q)\right)=M_{i}-1$, and let $Q_{*}=Q \cup B_{*}(Q)$, then $\rho\left(G^{*}\right)=Q_{*}$ and so by Theorem 3.2 and Observation 4.1 we get

$$
Q=\rho(G)=\rho\left(S_{u}\left(D\left(S_{d}\left(U\left(G^{*}\right)\right)\right)\right)\right)=S_{d}\left(U\left(S_{u}\left(D\left(\rho\left(G^{*}\right)\right)\right)\right)\right)=S_{d}\left(U\left(S_{u}\left(D\left(Q_{*}\right)\right)\right)\right) .
$$

Since $Q \subseteq U(\tilde{m}+\tilde{1}) \cap D(\tilde{M}-\tilde{1}) \subseteq \mathbb{N}^{d}$ is a finite set, we have by (6) the following dual theorem of Theorem 3.2 .

Theorem 4.2. For an antichain $Q=\left\{\tilde{p}_{1}, \ldots, \tilde{p}_{k}\right\} \subseteq \mathbb{N}^{d}$ we have

$$
Q=S_{d}\left(U\left(S_{u}\left(D\left(Q_{*}\right)\right)\right)\right)
$$

where $Q_{*}=Q \cup B_{*}(Q)$ and $B_{*}(Q)$ is as in (5). 
By the above Theorem 4.2 we see that given any antichain $Q \subseteq \mathbb{N}^{d}$, then the corresponding monomials are exactly the monomials in $\operatorname{\partial oc}(I(Q))$, where $I(Q)$ is the monomial ideal generated by the monomials that correspond to $S_{u}\left(D\left(Q_{*}\right)\right) \subseteq \mathbb{N}_{0}^{d}$. This does give a positive answer to one of our motivating questions in Section 1, Note, however, that this monomial ideal is not unique, as stated in the dual theorem of Theorem 4.3 here below.

Let $Q=\left\{\tilde{p}_{1}, \ldots, \tilde{p}_{k}\right\} \subseteq \mathbb{N}^{d}$ be an antichain and $\tilde{a} \leq \tilde{m}$ and $\tilde{b} \geq \tilde{M}$ where $\tilde{m}$ and $\tilde{M}$ are as in (5). Further, let $\rho=\rho_{\tilde{a}+\tilde{b}}$ as defined in the beginning of this section. If now $G=\rho(Q)$, then we have, as above, that $\rho(G)=\rho^{2}(Q)=Q$ and for $G^{*}(\tilde{a}, \tilde{b})$ as in the Remark right before Theorem 3.4, that

$$
\begin{aligned}
\rho\left(G^{*}(\tilde{a}, \tilde{b})\right) & =\rho\left(G \cup B^{*}(\tilde{a}, \tilde{b})\right) \\
& =\rho(G) \cup \rho\left(B^{*}(\tilde{a}, \tilde{b})\right) \\
& =Q \cup\left\{\left(a_{1}, b_{2}, b_{3} \ldots, b_{d}\right),\left(b_{1}, a_{2}, b_{3}, \ldots, b_{d}\right),\left(b_{1}, b_{2}, a_{3}, \ldots, b_{d}\right), \ldots,\left(b_{1}, b_{2}, b_{3}, \ldots, a_{d}\right)\right\} .
\end{aligned}
$$

So, again, we can define

$$
B_{*}(\tilde{a}, \tilde{b})=\left\{\left(a_{1}, b_{2}, b_{3} \ldots, b_{d}\right),\left(b_{1}, a_{2}, b_{3}, \ldots, b_{d}\right),\left(b_{1}, b_{2}, a_{3}, \ldots, b_{d}\right), \ldots,\left(b_{1}, b_{2}, b_{3}, \ldots, a_{d}\right)\right\} .
$$

If now

$$
Q_{*}(\tilde{a}, \tilde{b}):=Q \cup B_{*}(\tilde{a}, \tilde{b}),
$$

then $\rho\left(G^{*}(\tilde{a}, \tilde{b})\right)=Q_{*}(\tilde{a}, \tilde{b})$, and so by Theorem 3.4 and Observation 4.1 we get

$$
Q=\rho(G)=\rho\left(S_{u}\left(D\left(S_{d}\left(U\left(G^{*}(\tilde{a}, \tilde{b})\right)\right)\right)\right)\right)=S_{d}\left(U\left(S_{u}\left(D\left(\rho\left(G^{*}(\tilde{a}, \tilde{b})\right)\right)\right)\right)\right)=S_{d}\left(U\left(S_{u}\left(D\left(Q_{*}(\tilde{a}, \tilde{b})\right)\right)\right)\right) .
$$

Since this holds for any $\tilde{a} \leq \tilde{m}$ and $\tilde{b} \geq \tilde{M}$, we have a following dual theorem of Theorem 3.4 .

Theorem 4.3. For an antichain $Q=\left\{\tilde{p}_{1}, \ldots, \tilde{p}_{k}\right\} \subseteq \mathbb{N}^{d}$ and for $\tilde{a} \leq \tilde{m}$ and $\tilde{b} \geq \tilde{M}$ where $\tilde{m}$ and $\tilde{M}$ are as in (5), we have

$$
Q=S_{d}\left(U\left(S_{u}\left(D\left(Q_{*}(\tilde{a}, \tilde{b})\right)\right)\right)\right)
$$

where $Q_{*}(\tilde{a}, \tilde{b})=Q \cup B_{*}(\tilde{a}, \tilde{b})$ and $B_{*}(\tilde{a}, \tilde{b})$ is as in (7).

\section{Remarks.}

(i) Note that Theorem 4.3 is valid for any $\tilde{a}, \tilde{b} \in \mathbb{Z}^{d}$ that satisfy $\tilde{a} \leq \tilde{m}$ and $\tilde{b} \geq \tilde{M}$. This will be used in the next section.

(ii) Note that if $Q_{*}(\tilde{a}, \tilde{b})$ is given (without the prior knowledge of $B_{*}(\tilde{a}, \tilde{b})$ ), then we can retrieve $B_{*}(\tilde{a}, \tilde{b})$ as in in (7) from $Q_{*}(\tilde{a}, \tilde{b})$, and hence the set $Q$, if $\tilde{a} \leq \tilde{m}$ and $\tilde{b} \geq \tilde{M}$. 
(iii) Last but not least, note that by Observations 2.2 and 4.1, all Theorems 3.2, 3.4, 4.2 and 4.3 hold for any antichain $Q \subseteq \mathbb{Z}^{d}$, and not merely those of $\mathbb{N}^{d}$.

Example. Consider the case $d=3$ and the set $Q=\{(2,2,3),(3,3,2)\} \subseteq \mathbb{N}^{3}$. We use the above Theorem 4.2 to obtain an upset $U\left(S_{d}\left(D\left(Q_{*}\right)\right)\right.$ ), whose minimal points $S_{u}\left(D\left(Q_{*}\right)\right) \subseteq \mathbb{N}_{0}^{3}$ correspond to the generators of a monomial ideal $I$ where $\overline{\operatorname{Soc}(I)}$ is spanned by the image of $M=\left\{x^{2} y^{2} z^{3}, x^{3} y^{3} z^{2}\right\}$ in $R / I$, i.e. the monomials corresponding to the set $Q$, as follows.

By (5) we have here that $B_{*}(Q)=\{(4,4,1),(4,1,4),(1,4,4)\}$ and hence

$$
Q_{*}=Q \cup B_{*}(Q)=\{(2,2,3),(3,3,2),(4,4,1),(4,1,4),(1,4,4)\},
$$

and so $S_{u}\left(D\left(Q_{*}\right)\right)=\{(2,2,4),(2,3,3),(2,4,2),(3,2,3),(4,2,2)\}$. By Theorem 4.2 we now have

$$
\begin{aligned}
& S_{d}\left(U\left(S_{u}\left(D\left(Q_{*}\right)\right)\right)\right) \\
& \quad=S_{d}\left(U\left(S_{u}(D((2,2,3),(3,3,2),(4,4,1),(4,1,4),(1,4,4)))\right)\right) \\
& \quad=S_{d}(U((2,2,4),(2,3,3),(2,4,2),(3,2,3),(4,2,2))) \\
& \quad=\{(2,2,3),(3,3,2)\} \\
& =Q .
\end{aligned}
$$

Therefore, the monomial ideal $I_{1}=\left(x^{2} y^{2} z^{4}, x^{2} y^{3} z^{3}, x^{2} y^{4} z^{2}, x^{3} y^{2} z^{3}, x^{4} y^{2} z^{2}\right) \subseteq K[x, y, z]$ has $\overline{\operatorname{Soc}\left(I_{1}\right)}$ spanned by the image of $M=\left\{x^{2} y^{2} z^{3}, x^{3} y^{3} z^{2}\right\}$ in $R / I_{1}$ as a $k$-vector space.

Since $\tilde{a}=(0,0,1) \leq(1,1,1)=\tilde{m}$ and $\tilde{b}=(5,6,7) \geq(4,4,4)=\tilde{M}$ in $\mathbb{Z}^{3}$, we have for

$$
Q_{*}(\tilde{a}, \tilde{b})=Q_{*}((0,0,1),(5,6,7))=\{(2,2,3),(3,3,2),(5,6,1),(5,0,7),(0,6,7)\}
$$

by Theorem 4.3 that

$$
\begin{aligned}
& S_{d}\left(U\left(S_{u}\left(D\left(Q_{*}((0,0,1),(5,6,7))\right)\right)\right)\right) \\
& \quad=S_{d}\left(U\left(S_{u}(D((2,2,3),(3,3,2),(5,6,1),(5,0,7),(0,6,7)))\right)\right) \\
& =S_{d}(U((1,1,4),(1,3,3),(1,4,2),(3,1,3),(4,1,2))) \\
& =\{(2,2,3),(3,3,2)\} \\
& =Q .
\end{aligned}
$$

as well. Hence, the monomial ideal $I_{2}=\left(x y z^{4}, x^{1} y^{3} z^{3}, x y^{4} z^{2}, x^{3} y z^{3}, x^{4} y z^{2}\right) \subseteq K[x, y, z]$ also has $\overline{\operatorname{Soc}\left(I_{2}\right)}$ spanned by the image of $M=\left\{x^{2} y^{2} z^{3}, x^{3} y^{3} z^{2}\right\}$ in $R / I_{2}$ as a $k$-vector space. 


\section{Remarks.}

(i) As with many formulae, when it comes utilizing them to compute specific values, the compact forms and shortness is not always a guarantee for a fast evaluation. Conversely, a seemingly ugly expression can many times be much better to use to obtain specific values in a fast and an efficient manner. The computation of $S_{u}\left(D\left(Q_{*}\right)\right)$, from an antichain $Q$ consisting of $k$ points from $\mathbb{N}^{d}$ as in Theorem 4.2, can for each fixed $k$ be done in polynomial time in $k$ alone. In fact, it can be done in $O\left(k^{d}\right)$-time, although the exact or a tight upper bound of its complexity is hard to come by.

(ii) We have so far assumed $G, Q \subseteq \mathbb{N}^{d}$, only to guarantee that $G^{*}, Q_{*} \subseteq \mathbb{N}_{0}^{d}$. However, general $G, Q \subseteq \mathbb{N}_{0}^{d}$ will imply $G^{*}, Q_{*} \subseteq\{-1,0,1,2, \ldots\}^{d} \subseteq \mathbb{Z}^{d}$ which does not correspond to a set of monomials from $\left[x_{1}, \ldots, x_{d}\right]$ but rather monomials from $\left[x_{1}, x_{1}^{-1}, \ldots, x_{d}, x_{d}^{-1}\right]$.

\section{ZERO-DIMENSIONAL MONOMIAL IDEALS}

For a field $K$, the ring $R=K\left[x_{1}, \ldots, x_{d}\right]$, the maximal ideal $\mathbf{m}$ of $R$ and a monomial ideal $I$ of $R$, the motivation for this section is the following question.

Question 5.1. Under what circumstances can we retrieve a unique monomial ideal $I$ from the monomials of $\operatorname{Soc}(I)=\operatorname{Soc}_{\mathbf{m}}(I)$ that are not in $I$ ?

We saw in the last example in the previous Section 4 that both the following ideals of $R=$ $K[x, y, z]$

$$
\begin{aligned}
& I_{1}=\left(x^{2} y^{2} z^{4}, x^{2} y^{3} z^{3}, x^{2} y^{4} z^{2}, x^{3} y^{2} z^{3}, x^{4} y^{2} z^{2}\right) \text { and } \\
& I_{2}=\left(x y z^{4}, x^{1} y^{3} z^{3}, x y^{4} z^{2}, x^{3} y z^{3}, x^{4} y z^{2}\right)
\end{aligned}
$$

have $\overline{\operatorname{Soc}\left(I_{1}\right)}$ and resp. $\overline{\operatorname{Soc}\left(I_{2}\right)}$ spanned by the image of $M=\left\{x^{2} y^{2} z^{3}, x^{3} y^{3} z^{2}\right\}$ in $R / I_{1}$ and resp. $R / I_{2}$ as a $k$-vector space. So Question 5.1 has in general a negative answer. In fact, for any monomial ideal $I$ it is easy to construct a rich family of monomial ideals such that $\partial \mathrm{oc}\left(I^{\prime}\right)=\partial \mathrm{oc}(I)$ for any ideal $I^{\prime}$ in that family.

If, however, we assume $\operatorname{dim}(I)=0$ then, we will see shortly, Question 5.1 has a positive answer. Zero-dimensional monomial ideals of $R=K\left[x_{1}, \ldots, x_{d}\right]$ constitute an interesting yet fairly general class of monomial ideals for numerous reasons: the quotient ring $R / I$ is a local ring with a unique prime and maximal ideal, $R / I$ is finite dimensional over $k$, and their variety consist of a single point $\tilde{0}$, to name a few. 
For a zero-dimensional ideal $I \neq \mathbf{m}$ of $R$ we note that the image of $\operatorname{Soc}_{\mathbf{m}}(I)$ in $R / I$ corresponds to an antichain $Q$ of $\mathbb{N}_{0}^{d}$. As mentioned in the remark after Theorem 4.3 , we then have for our $Q \subseteq \mathbb{N}_{0}^{d}$ that $Q=S_{d}\left(U\left(S_{u}\left(D\left(Q_{*}(\tilde{a}, \tilde{b})\right)\right)\right)\right)$ for any suitable $\tilde{a}, \tilde{b} \in \mathbb{Z}^{d}$. In particular, since we have $-\tilde{1}=$ $(-1, \ldots,-1) \leq \tilde{m}$ from Theorem 4.3 , then for any $\tilde{b} \geq \tilde{M}$ we have $Q=S_{d}\left(U\left(S_{u}\left(D\left(Q_{*}(-\tilde{1}, \tilde{b})\right)\right)\right)\right)$. We now briefly argue that the upset $U\left(S_{u}\left(D\left(Q_{*}(-\tilde{1}, \tilde{b})\right)\right)\right)$ corresponds to a monomial ideal $I$ of $R$ of dimension zero: first we note that by definition (7) we have

$$
\begin{aligned}
D\left(Q_{*}(-\tilde{1}, \tilde{b})\right) & \supseteq D\left(B_{*}(-\tilde{1}, \tilde{b})\right) \\
& =D\left(\left(-1, b_{2}, b_{3} \ldots, b_{d}\right),\left(b_{1},-1, b_{3}, \ldots, b_{d}\right), \ldots,\left(b_{1}, b_{2}, b_{3}, \ldots,-1\right)\right.
\end{aligned}
$$

and hence $S_{u}\left(D\left(Q_{*}(-\tilde{1}, \tilde{b})\right)\right) \subseteq U(\tilde{0})=\mathbb{N}_{0}^{d}$, and so the generators for the upset $U\left(S_{u}\left(D\left(Q_{*}(-\tilde{1}, \tilde{b})\right)\right)\right)$ correspond to monomials of $R=K\left[x_{1}, \ldots, x_{d}\right]$. Secondly, since $\tilde{b} \geq \tilde{M}$ as in Theorem 4.3, then for each $i \in[d]$ we have $b_{i} \tilde{e}_{i}=\left(0, \ldots, 0, b_{i}, 0, \ldots, 0\right) \notin D\left(Q_{*}(-\tilde{1}, \tilde{b})\right)$ and $-\tilde{e}_{i}=(0, \ldots, 0,-1,0, \ldots, 0) \in$ $D\left(Q_{*}(-\tilde{1}, \tilde{b})\right)$, and so there is a smallest $c_{i} \in \mathbb{N}_{0}, c_{i} \leq b_{i}$ with $c_{i} \tilde{e}_{i} \notin D\left(Q_{*}(-\tilde{1}, \tilde{b})\right)$. Since $c_{i} \tilde{e}_{i}-\tilde{e}_{j} \in$ $D\left(Q_{*}(-\tilde{1}, \tilde{b})\right)$ for each $j \neq i$ and $c_{i} \tilde{e}_{i}-\tilde{e}_{i}=\left(c_{i}-1\right) \tilde{e}_{i} \in D\left(Q_{*}(-\tilde{1}, \tilde{b})\right)$, we have that $c_{i} \tilde{e}_{i} \in$ $S_{u}\left(D\left(Q_{*}(-\tilde{1}, \tilde{b})\right)\right)$ for each $i$, which means that $x_{i}^{c_{i}}$ is an element of the monomial ideal of $I$ that corresponds to $U\left(S_{u}\left(D\left(Q_{*}(-\tilde{1}, \tilde{b})\right)\right)\right)$, showing that $I$ is indeed of dimension zero.

To see that the zero-dimensional ideal $I$ is unique, it suffices to show that the upset from above $U=U\left(S_{u}\left(D\left(Q_{*}(-\tilde{1}, \tilde{b})\right)\right)\right)$ is the unique upset with $Q=S_{d}(U)$. Assume $I_{1}$ and $I_{2}$ are two zerodimensional monomial ideals with the same socle w.r.t. the maximal ideal $\mathbf{m}$ of $R$. As each $I_{i}$ is zero-dimensional, it corresponds to an upset $U\left(G_{i}^{*}\left(\tilde{0}, \tilde{b}_{i}\right)\right)$ where $G_{i}^{*}\left(\tilde{0}, \tilde{b}_{i}\right)=G_{i} \cup B^{*}\left(\tilde{0}, \tilde{b}_{i}\right)$ is an antichain of $\mathbb{N}_{0}^{d}$ as in (44). By our assumption we have $S_{d}\left(U\left(G_{1}^{*}\left(\tilde{0}, \tilde{b}_{1}\right)\right)\right)=Q=S_{d}\left(U\left(G_{2}^{*}\left(\tilde{0}, \tilde{b}_{2}\right)\right)\right)$ and hence by Theorem 3.4 we then get

$$
G_{1}=S_{u}\left(D\left(S_{d}\left(U\left(G_{1}^{*}\left(\tilde{0}, \tilde{b}_{1}\right)\right)\right)\right)\right)=S_{u}(D(Q))=S_{u}\left(D\left(S_{d}\left(U\left(G_{1}^{*}\left(\tilde{0}, \tilde{b}_{1}\right)\right)\right)\right)\right)=G_{2} .
$$

Also, directly from $S_{d}\left(U\left(G_{1}^{*}\left(\tilde{0}, \tilde{b}_{1}\right)\right)\right)=Q=S_{d}\left(U\left(G_{2}^{*}\left(\tilde{0}, \tilde{b}_{2}\right)\right)\right)$, we obtain for each $i \in[d]$ that

$$
b_{1 i}=\pi_{i}\left(\tilde{b}_{1}\right)=\max \left(\left\{\pi_{i}(\tilde{q}): \tilde{q} \in Q\right\}\right)=\pi_{i}\left(\tilde{b}_{2}\right)=b_{12},
$$

and hence $\tilde{b}_{1}=\tilde{b}_{2}$. Therefore

$$
G_{1}^{*}\left(\tilde{0}, \tilde{b}_{1}\right)=G_{1} \cup B^{*}\left(\tilde{0}, \tilde{b}_{1}\right)=G_{2} \cup B^{*}\left(\tilde{0}, \tilde{b}_{2}\right)=G_{2}^{*}\left(\tilde{0}, \tilde{b}_{2}\right),
$$

and so $U\left(G_{1}^{*}\left(\tilde{0}, \tilde{b}_{1}\right)=U\left(G_{2}^{*}\left(\tilde{0}, \tilde{b}_{2}\right)\right.\right.$. This means that the corresponding zero-dimensional monomial ideals are equal $I_{1}=I_{2}$. This yields a positive answer to Question 5.1 for zero-dimensional monomial 
ideals, as stated in the following proposition, in which the set $S$ of monomials corresponds to our antichain $Q \subseteq \mathbb{N}_{0}^{d}$ from above.

Proposition 5.2. For any non-empty set $S$ of non-comparable monomials of $R=K\left[x_{1}, \ldots, x_{d}\right]$ there is a unique zero-dimensional ideal $I$ of $R$ with $\partial \mathrm{oc}(I)=S$.

For the remainder of this section we discuss in further detail the cases when the set $S$ of monomials in Proposition 5.2, that corresponds to an antichain $Q \subseteq \mathbb{N}_{0}^{d}$, has cardinality 1 and 2, that is $|S|=|Q| \in\{1,2\}$.

The case when $Q$ is a singleton. An interesting case of Proposition 5.2 this is when $|Q|=1$, so the antichain $Q$ of $\mathbb{N}_{0}^{d}$ consists of just one point, say $Q=\{\tilde{p}\}$. As before, the unique zerodimensional monomial ideal corresponds to the upset $U=U\left(S_{u}\left(D\left(Q_{*}(-\tilde{1}, \tilde{b})\right)\right)\right)$ where $Q=S_{d}(U)$. If now $\tilde{p}=\left(p_{1}, \ldots, p_{d}\right) \in \mathbb{N}_{0}^{d}$, we note that the downset $D\left(Q_{*}(-\tilde{1}, \tilde{b})\right)$ is generated by an antichain consisting of $d+1$ elements

$$
Q_{*}(-\tilde{1}, \tilde{b})=\left\{\tilde{p},\left(-1, b_{2}, b_{3} \ldots, b_{d}\right),\left(b_{1},-1, b_{3}, \ldots, b_{d}\right), \ldots,\left(b_{1}, b_{2}, b_{3}, \ldots,-1\right)\right\} .
$$

Since each element $\tilde{r} \in S_{u}\left(D\left(Q_{*}(-\tilde{1}, \tilde{b})\right)\right)$ is, by definition of $S_{u}(D)$, uniquely determined by $d$ distinct elements of $Q_{*}(-\tilde{1}, \tilde{b})$ (by the conditions (i) $\tilde{r} \notin Q_{*}(-\tilde{1}, \tilde{b})$ and (ii) $\tilde{r}-\tilde{e}_{i} \in Q_{*}(-\tilde{1}, \tilde{b})$ for each $i \in[d])$, we have that $S_{u}\left(D\left(Q_{*}(-\tilde{1}, \tilde{b})\right)\right)$ is a subset of the possible $\left(\begin{array}{c}d+1 \\ d\end{array}\right)=d+1$ elements $\left\{\tilde{0},\left(p_{1}+1\right) \tilde{e}_{1}, \ldots,\left(p_{d}+1\right) \tilde{e}_{d}\right\}$, and hence, as it is an antichain, we obtain

$$
\left.S_{u}\left(D\left(Q_{*}(-\tilde{1}, \tilde{b})\right)\right)=\left\{\left(p_{1}+1\right) \tilde{e}_{1}, \ldots,\left(p_{d}+1\right) \tilde{e}_{d}\right\}\right\},
$$

which means that the upset $U=U\left(S_{u}\left(D\left(Q_{*}(-\tilde{1}, \tilde{b})\right)\right)\right)$ corresponds to a monomial ideal $I$ of the form $I=\left(x_{1}^{p_{1}+1}, \ldots, x_{d}^{p_{d}+1}\right)$.

That $|S|=1$ means that $\partial \mathrm{oc}(I)=S$ contains the unique generator of the ideal $\overline{\operatorname{Soc}_{\mathbf{m}}(I)}$ of $R / I$. This means that $R / I$ is a zero dimensional local Gorenstein ring [Eis95, Prop. 21.5]. Clearly, if our monomial ideal $I$ is generated by pure powers of the variables $x_{1}, \ldots, x_{d}$, then $R / I$ is a zero dimensional local Gorenstein ring with a unique monomial in $\partial \mathrm{oc}(I)$. Hence, we obtain as a corollary the following description of Artinian Gorenstein rings that are defined by monomials [Bei93], see also Wol16].

Corollary 5.3. Let $R=K\left[x_{1}, \ldots, x_{d}\right]$ and $I$ be a monomial ideal of $R$. Then $R / I$ is a zero dimensional local Gorenstein ring if, and only if, $I=\left(x_{1}^{p_{1}+1}, \ldots, x_{d}^{p_{d}+1}\right)$ for some $\tilde{p} \in \mathbb{N}_{0}^{d}$, in which case $\operatorname{\partial oc}(I)=\left\{\tilde{x}^{\tilde{p}}=x_{1}^{p_{1}} \cdots x_{d}^{p_{d}}\right\}$. 
For a general $Q \subseteq \mathbb{Z}^{d}$ we have, as mentioned here above, that every element of $S_{u}(D(Q))$ is uniquely determined by $d$ distinct elements of $Q$. More specifically we have the following.

Claim 5.4. For an antichain $G \subseteq \mathbb{Z}^{d}$ and $\tilde{r} \in S_{d}(U(G))$ there are distinct $\tilde{p}_{1}, \ldots, \tilde{p}_{d} \in G$ such that (i) $\tilde{r} \in S_{d}\left(U\left(\tilde{p}_{1}, \ldots, \tilde{p}_{d}\right)\right)$ and (ii) $\tilde{r}=\left(p_{11}+1, \ldots, p_{d d}+1\right)$.

Dually, for an antichain $Q \subseteq \mathbb{Z}^{d}$ and $\tilde{r} \in S_{u}(D(Q))$ there are distinct $\tilde{p}_{1}, \ldots, \tilde{p}_{d} \in Q$ such that (i) $\tilde{r} \in S_{d}\left(U\left(\tilde{p}_{1}, \ldots, \tilde{p}_{d}\right)\right)$ and (ii) $\tilde{r}=\left(p_{11}-1, \ldots, p_{d d}-1\right)$.

We can in similar fashion, as for Corollary [5.3, consider the case when $|S|=k$, that is the corresponding antichain $Q$ of $\mathbb{Z}_{0}^{d}$ contains $k$ points $Q=\left\{\tilde{p}_{1}, \ldots, \tilde{p}_{k}\right\}$, where each $\tilde{p}_{i}=\left(p_{i 1}, \ldots, p_{i d}\right)$. In this case we have $Q_{*}(-\tilde{1}, \tilde{b})=Q \cup B_{*}(-\tilde{1}, \tilde{b})$ where $B_{*}(-\tilde{1}, \tilde{b})$ is as in (17), and hence $b_{i} \geq$ $\max \left(p_{i 1}+1, \ldots, p_{i d}+1\right)$ for each index $i$, and so the downset $D\left(Q_{*}(-\tilde{1}, \tilde{b})\right)$ is generated by an antichain of $d+k$ elements. For convenience we let

$$
\tilde{b}(i):=\left(b_{1}, \ldots, b_{i-1},-1, b_{i+1}, \ldots, b_{d}\right),
$$

for each $i \in[d]$, so $B_{*}(-\tilde{1}, \tilde{b})=\{\tilde{b}(1), \ldots, \tilde{b}(d)\}$. The following lemma provides a useful tool.

Lemma 5.5. For $\mathcal{I}=\left\{i_{1}, \ldots i_{k}\right\} \subseteq[d]$ and $D=D\left(\tilde{p}_{1}, \ldots, \tilde{p}_{k}, \tilde{b}(1), \ldots, \widetilde{b}\left(i_{1}\right), \ldots, \widetilde{b}\left(i_{k}\right), \ldots, \tilde{b}(d)\right)$ we have

$$
S_{u}(D)= \begin{cases}\left\{\sum_{\ell=1}^{k} r_{i_{\ell}} \tilde{e}_{i_{\ell}}\right\} & \text { if } S_{u}\left(D\left(\pi_{I}\left(\tilde{p}_{1}\right), \ldots, \pi_{I}\left(\tilde{p}_{k}\right)\right)\right)=\left\{\left(r_{i_{1}}, \ldots, r_{i_{k}}\right)\right\} \neq \emptyset \\ \emptyset & \text { otherwise. }\end{cases}
$$

Proof. For $\tilde{r} \in S_{u}(D)$ we have by definition that $\tilde{r} \notin D$ and $\tilde{r}-\tilde{e}_{\ell} \in D$ for each $\ell$. Since $D \subseteq D(\tilde{b})$ we have then $r_{k} \leq b_{k}$ for each $k \neq \ell$. As this holds for each $\ell$ we have $\tilde{r} \leq \tilde{b}$.

Further, for $\tilde{r} \in S_{u}(D)$, there is, analogous to Claim [5.4 a permutation $\sigma \in S_{d}$ such that $\tilde{r}-\tilde{e}_{\sigma(\ell)} \leq \tilde{b}(\ell)$ for each index $\ell \notin \mathcal{I}$ and $\tilde{r}-\tilde{e}_{\sigma\left(i_{\ell}\right)} \leq \tilde{p}_{\ell}$ for $1 \leq \ell \leq k$. Since $\tilde{r} \notin D$ we hence have for each $\ell \notin \mathcal{I}$ that

$$
r_{\sigma(\ell)}= \begin{cases}b_{\sigma(\ell)}+1 & \text { if } \sigma(\ell) \neq \ell \\ 0 & \text { if } \sigma(\ell)=\ell\end{cases}
$$

and $r_{\sigma(\ell)}=p_{\ell \sigma(\ell)}+1$ for each $\ell \in \mathcal{I}$. Since $\tilde{r} \leq \tilde{b}$ we must by (8) have that $r_{\sigma(\ell)}=0$ for each $\ell \notin \mathcal{I}$ and therefore $\sigma(\ell)=\ell$ for each $\ell \notin \mathcal{I}$. Hence we have $r_{\ell}=0$ for each $\ell \notin \mathcal{I}$. Consequently $\sigma \in S(\mathcal{I})$ is a permutation only on $\mathcal{I}$ and leaves every other element of $[d] \backslash \mathcal{I}$ fixed. In particular we have that

$$
\tilde{r}=\sum_{\ell=1}^{k} r_{i_{\ell}} \tilde{e}_{i_{\ell}}=\sum_{i \in \mathcal{I}} r_{i} \tilde{e}_{i}
$$


Since $\tilde{r} \notin D$ we have $\tilde{r} \notin D\left(\tilde{p}_{i_{1}}, \ldots, \tilde{p}_{i_{k}}\right)$ and since $\tilde{r}-\tilde{e}_{\sigma\left(i_{\ell)}\right.} \leq \tilde{p}_{\ell}$ for each $\ell \in[k]$, or $\tilde{r}-\tilde{e}_{\ell} \leq$ $\tilde{p}_{\gamma(\ell)}$ for each $\ell \in \mathcal{I}$, where $\gamma: \mathcal{I} \rightarrow[k]$ is the map $i_{\ell} \mapsto \ell$, we have $\left(r_{i_{1}}, \ldots, r_{i_{k}}\right)=\pi_{\mathcal{I}}(\tilde{r}) \in$ $S_{u}\left(D\left(\pi_{\mathcal{I}}\left(\tilde{p}_{1}\right), \ldots, \pi_{\mathcal{I}}\left(\tilde{p}_{k}\right)\right)\right)$, which by Claim 5.4 is uniquely determined.

From the above proof we note that if $S_{u}(D) \neq \emptyset$ and $\tilde{r} \in S_{u}(D)$, then $r_{\sigma\left(i_{\ell}\right)}=P_{\ell \sigma\left(i_{\ell}\right)}+1$ for $1 \leq \ell \leq k$ and so $r_{\sigma\left(i_{\ell}\right)}=P_{\gamma\left(i_{\ell}\right) \sigma\left(i_{\ell}\right)}+1$ for $1 \leq \ell \leq k$, or $r_{\ell}=p_{\beta(\ell) \ell}+1$ for each $\ell \in \mathcal{I}$, where $\beta=\gamma \circ \sigma^{-1}$, and so

$$
\tilde{r}=\left(p_{\beta\left(i_{i}\right) i_{1}}+1\right) \tilde{e}_{i_{1}}+\cdots+\left(p_{\beta\left(i_{k}\right) i_{k}}+1\right) \tilde{e}_{i_{k}}
$$

for the bijection $\beta: \mathcal{I} \rightarrow[k]$. From this we get the following.

Observation 5.6. Let $\mathcal{I}=\left\{i_{1}, \ldots i_{k}\right\} \subseteq[d]$ and $D=D\left(\tilde{p}_{1}, \ldots, \tilde{p}_{k}, \tilde{b}(1), \ldots, \widetilde{b\left(i_{1}\right)}, \ldots, \widehat{\tilde{b}\left(i_{k}\right)}, \ldots, \tilde{b}(d)\right)$. If $S_{u}(D) \neq \emptyset$ and $\tilde{r} \in S_{u}(D)$ is its unique element, then there is a permutation $\alpha \in S_{k}$ such that

$$
\pi_{\mathcal{I}}(\tilde{r})=\left(p_{\alpha(1) i_{1}}+1, \ldots, p_{\alpha(k) i_{k}}+1\right) \in S_{u}\left(D\left(\pi_{\mathcal{I}}\left(\tilde{p}_{1}\right), \ldots, \pi_{\mathcal{I}}\left(\tilde{p}_{k}\right)\right)\right)
$$

The case when $k=|Q|=2$. The second special case of $k=2$, so $Q=\{\tilde{p}, \tilde{q}\}$, is, as we will see here below, simple enough so that we can state some conclusion in a direct and uncluttered manner. The following follows directly from the above Lemma 5.5 and Observation 5.6 .

Corollary 5.7. For two distinct indices $i, j \in[d]$ and $D=D(\tilde{p}, \tilde{q}, \tilde{b}(1), \ldots, \widehat{\tilde{b}(i)}, \ldots, \widehat{\tilde{b}(j)}, \ldots, \tilde{b}(d))$ we have

$$
S_{u}(D)= \begin{cases}\left\{\left(p_{i}+1\right) \tilde{e}_{i}+\left(q_{j}+1\right) \tilde{e}_{j}\right\} & \text { if } p_{i}<q_{i} \text { and } q_{j}<p_{j} \\ \left\{\left(q_{i}+1\right) \tilde{e}_{i}+\left(p_{j}+1\right) \tilde{e}_{j}\right\} & \text { if } q_{i}<p_{i} \text { and } p_{j}<q_{j} \\ \emptyset & \text { otherwise. }\end{cases}
$$

For the antichain $Q=\{\tilde{p}, \tilde{q}\}$ of $\mathbb{N}_{0}^{d}$ we have a pseudo-partition $[d]=A \cup B \cup C$ (some parts could be empty) where $p_{i}<q_{i}$ for all $i \in A, p_{i}=q_{i}$ for all $i \in B$, and $p_{i}>q_{i}$ for all $i \in C$. We have here that

$$
Q_{*}(-\tilde{1}, \tilde{b})=Q \cup B_{*}(-\tilde{1}, \tilde{b})=\{\tilde{p}, \tilde{q}, \tilde{b}(1), \ldots, \tilde{b}(d)\}
$$

Since $S_{u}(D)$ forms an antichain, for any downset $D \subseteq \mathbb{Z}^{d}$, and each element of $S_{u}\left(Q_{*}(-\tilde{1}, \tilde{b})\right.$ Is uniquely determined by a $d$-subset of $Q_{*}(-\tilde{1}, \tilde{b})$, then $S_{u}\left(Q_{*}(-\tilde{1}, \tilde{b})\right.$ is among the at most $\left(\begin{array}{c}d+2 \\ d\end{array}\right)=$ $\left(\begin{array}{c}d+2 \\ 2\end{array}\right)$ elements of $\bigcup_{Q^{\prime}} S_{u}\left(D\left(Q^{\prime}\right)\right)$, where the union is taken over all $Q^{\prime} \subseteq Q_{*}(-\tilde{1}, \tilde{b})$ with cardinality d. Since $[d]=A \cup B \cup C$ is a pseudo-partition, then by letting $|A|=\mathbf{a},|B|=\mathbf{b}$ and $|C|=\mathbf{c}$ we have $|A|+|B \cup C|=\mathbf{a}+\mathbf{b}+\mathbf{c}=d$, and therefore, by Corollary 5.7, we then have the following. 
Proposition 5.8. For an antichain $Q=\{\tilde{p}, \tilde{q}\}$ of of $\mathbb{Z}_{0}^{d}$ and the corresponding pseudo-partition $[d]=A \cup B \cup C$ we have

$$
\begin{aligned}
& S_{u}\left(D\left(Q_{*}(-\tilde{1}, \tilde{b})\right)\right)= \\
& \quad\left\{\left(p_{i}+1\right) \tilde{e}_{i}+\left(q_{j}+1\right) \tilde{e}_{j}:(i, j) \in A \times C\right\} \cup\left\{\left(p_{i}+1\right) \tilde{e}_{i}: i \in B \cup C\right\} \cup\left\{\left(q_{i}+1\right) \tilde{e}_{i}: i \in A\right\},
\end{aligned}
$$

and hence $\left|S_{u}\left(D\left(Q_{*}(-\tilde{1}, \tilde{b})\right)\right)\right|=\mathbf{a c}+d$.

Note that

$$
\begin{aligned}
& \left\{\left(p_{i}+1\right) \tilde{e}_{i}: i \in B \cup C\right\} \cup\left\{\left(q_{i}+1\right) \tilde{e}_{i}: i \in A\right\} \\
= & \left\{\left(p_{i}+1\right) \tilde{e}_{i}: i \in B \cup C\right\} \cup\left\{\left(q_{i}+1\right) \tilde{e}_{i}: i \in A \cup B\right\} \\
= & \left\{\left(p_{i}+1\right) \tilde{e}_{i}: i \in C\right\} \cup\left\{\left(q_{i}+1\right) \tilde{e}_{i}: i \in A \cup B\right\} .
\end{aligned}
$$

In the above display, the first and the last unions are pseudo-partitions and not symmetric, whereas the middle union is symmetric but not disjoint.

Similar to Corollary 5.3 describing Artinian Gorenstein rings defined by monomial from [Bei93], we can interpret the above Proposition 5.8 for Artinian rings defined by monomials that are almost Gorenstein as defined in [HV06] as those monomial ideals $I$ of $R=K\left[x_{1}, \ldots, x_{d}\right]$ with $\partial \mathrm{oc}(I)=S$ from Proposition 5.2 being a set of "small" cardinality. More specifically, if $I$ is a zero dimensional monomial ideal of $R$ with $|S|=k \geq 1$, then $I$ is said to be of type $k$, and so type 1 zero dimensional ideals are exactly the Artinian Gorenstein monomial ideals [HV06. The above Proposition 5.8 gives a complete description of the type 2 zero dimensional monomial ideals of $R$.

Corollary 5.9. Let $R=K\left[x_{1}, \ldots, x_{d}\right]$ and $I$ be a monomial ideal of $R$. Then $R / I$ is a zero dimensional local type 2 ring if, and only if, for two incomparable $\tilde{p}, \tilde{q} \in \mathbb{Z}_{0}^{d}$ with corresponding pseudo-partition $[d]=A \cup B \cup C$ we have

$$
I=\left(x_{i}^{p_{i}+1} x_{j}^{q_{j}+1}, x_{h}^{p_{h}+1}, x_{k}^{q_{k}+1}:(i, j) \in A \times C, h \in B \cup C, k \in A\right),
$$

in which case $\partial \mathrm{oc}(I)=S=\left\{\tilde{x}^{\tilde{p}}, \tilde{x}^{\tilde{q}}\right\}$.

\section{Remarks.}

(i) Note that Corollary 5.9 characterizes completely those monomial ideals $I$ of $R=K\left[x_{1}, \ldots, x_{d}\right]$ that make $R / I$ a local Artinian ring of type 2, in a purely combinatorial way, using only the poset structure of $\mathbb{Z}^{d}$, 
(ii) Comparing with [HV06, Example 4.3] we see that the analysis of type 2 monomial ideal done there is slightly different from ours from Corollary 5.9, in that there in [HV06] the authors describe when exactly the condition $J_{1}: J_{2}+J_{2}: J_{1} \supseteq \mathbf{m}=\left(x_{1}, \ldots, x_{d}\right)$ from [HV06, Theorem 4.2] holds, where $I=J_{1} \cap J_{2}$ is the irredundant intersection of two irreducible ideals $J_{1}$ and $J_{2}$, each of which must be monomial ideals generated by powers of the variables $x_{1}, \ldots, x_{d}$

\section{Almost Gorenstein monomial ideals with $k \geq 3$}

The general case for $k=|Q| \geq 3$. We have so far analysed fully the structure of the antichain $S_{u}\left(Q_{*}(-\tilde{1}, \tilde{b})\right)$ of $\mathbb{Z}_{0}^{d}$ in the cases when $k=|Q| \in\{1,2\}$, the antichain corresponding to the generators of a zero-dimensional monomial ideal with $\partial \mathrm{oc}(I)$ corresponding to $Q$. These two cases are unique in that the form of the antichain $Q$ is quite simple: an antichain $Q=\{\tilde{p}, \tilde{q}\}$ in $\mathbb{Z}^{d}$ with the corresponding pseudo-partition $[d]=A \cup B \cup C$ as indicated in Proposition 5.8 must have both

parts $A$ and $C$ non-empty and so ac $\geq 1$. This means that the parts (here $A$ and $C$ ) corresponding to the two possible strict orderings of the coordinates, namely $p_{i}<q_{i}$ or $p_{i}>q_{i}$, are each nonempty. Needless to say, for an antichain $Q \subseteq \mathbb{Z}^{d}$ where $k=|Q| \geq 3$, this need not be case. The possibilities are simply to great. There are plenty antichains (in fact, vastly most of them, as we shall see momentarily) where not all strict orderings of the coordinates are present.

Analogous to the pseudo-partition $[d]=A \cup B \cup C$ from Proposition 5.8 we have for general $k \in \mathbb{N}$ a pseudo-partition

$$
[d]=\bigcup_{\omega \in W(k)} A_{\omega}
$$

where $W(k)$ is the collection of weak orderings of the set $[k]$ : the collection of distinct orderings of the elements where equality is allowed. In particular we clearly have for each $k \geq 2$ that $|W(k)|>k$ !, the number of strict orderings of $[k]$, and so the union in (9) is large. More specifically, for $k=1,2$ and 3 there are 1,3 and 13 weak orderings in $W(k)$ respectively, and, in general, $a(k)=|W(k)|$ is the $k$-th ordered Bell number or Fubini number given explicitly by

$$
a(k)=\sum_{i=1}^{k}\left\{\begin{array}{l}
k \\
i
\end{array}\right\} i !
$$


where $\left\{\begin{array}{l}k \\ i\end{array}\right\}=S(k, i)$ is the Stirling number of the second kind Sti] and where the exponential generating function for the corresponding sequence $(a(k))_{k \geq 0}=(1,1,3,13,75,541, \ldots)$ is given by

$$
\sum_{k=0}^{\infty} \frac{a(k)}{k !} x^{k}=\frac{1}{2-e^{x}} .
$$

Asymptotically $a(k) \approx \frac{n !}{2(\log 2)^{n+1}}=\frac{1}{2}(1.4427 \cdots)^{n+1} n$ !, where $\log$ is the natural logarithm and so $\log 2<1$, and so we see that the number $k$ ! of strict orderings of $[k]$ constitutes only a small fraction of the $a(k)$ weak orderings of $[k]$. The ordered Bell numbers have been studied extensively $\mathrm{OBn}$, A00.

Definition 6.1. An antichain $Q=\left\{\tilde{p}_{1}, \ldots, \tilde{p}_{k}\right\} \subseteq \mathbb{Z}^{d}$ is called order-generic if in the corresponding pseudo-partition (9) for each $\omega \in W(k)$ of strict orderings of $[k]$ we have $A_{\omega} \neq \emptyset$.

Note that in order for an antichain $Q \subseteq \mathbb{Z}^{d}$ to be order-generic we must have $d \geq k$ !. We will see that the structure of the antichain $S_{u}\left(Q_{*}(-\tilde{1}, \tilde{b})\right)$ of $\mathbb{Z}_{0}^{d}$ when $Q$ is order-generic is nice enough for some specific enumerations.

The following slight reformulation follows directly from Lemma 5.5 and Observation 5.6 .

Corollary 6.2. For $\mathcal{I}=\left\{i_{1}, \ldots i_{k}\right\} \subseteq[d]$ containing distinct elements and

$$
D=D\left(\tilde{p}_{1}, \ldots, \tilde{p}_{k}, \tilde{b}(1), \ldots, \widehat{b}\left(\widehat{\left.i_{1}\right)}, \ldots, \widehat{\tilde{b}\left(i_{k}\right)}, \ldots, \tilde{b}(d)\right)\right.
$$

we have

$$
S_{u}(D)=\left\{\left(p_{\alpha(1) i_{1}}+1\right) \tilde{e}_{i_{1}}+\cdots+\left(p_{\alpha(k) i_{k}}+1\right) \tilde{e}_{i_{k}}\right\},
$$

if there is a unique permutation $\alpha \in S_{k}$ with $p_{\alpha(h) i_{h}}<p_{\alpha(1) i_{h}}, \ldots, \widehat{p_{\alpha(h) i_{h}}}, \ldots, p_{\alpha(k) i_{h}}$ for each $h \in[k]$ and $S_{u}(D)=\emptyset$ otherwise.

For $k \in \mathbb{N}$ we have that $S_{u}\left(Q_{*}(-\tilde{1}, \tilde{b})\right.$, the set of $\mathbb{N}_{0}^{d}$ corresponding to the generators of our desired monomial ideal, is the antichain formed by the maximal elements of all the $\left(\begin{array}{c}d+k \\ d\end{array}\right)=\left(\begin{array}{c}d+k \\ k\end{array}\right)$ possible singletons $S_{u}(D)$, where $D \subseteq Q_{*}(-\tilde{1}, \tilde{b})$ contains exactly $d$ elements, and hence there are $k$ types of points in $S_{u}\left(Q_{*}(-\tilde{1}, \tilde{b})\right.$ : for each $\ell \in[k]$ the ones that are formed by a $d$-set $D$ that contains exactly $\ell$ points from $Q=\left\{\tilde{p}_{1}, \ldots, \tilde{p}_{k}\right\}$. Since our antichain is order-generic each mentioned type occurs in $S_{u}\left(Q_{*}(-\tilde{1}, \tilde{b})\right.$. To effectively list of these points we make the following convention. For each $a \in[k]$ and a subset $C \subseteq[k]$ we let

$$
B_{a}(C):=\left\{i \in[d]: p_{a i}<p_{b i} \text { for all } b \in C \backslash\{a\}\right\}
$$


For $C \subseteq C^{\prime} \subseteq[k]$ we clearly have $B_{a}\left(C^{\prime}\right) \subseteq B_{a}(C)$. With this convention the sets of the mentioned type $\ell \in[k]$, that contain those points formed by $d$-set $D$ containing $\ell$ points from $Q$, are those labeled by a $C \subseteq[k]$ with $|C|=\ell$, and are of the form

$$
P_{C}=\left\{\sum_{t \in C}\left(p_{t i_{t}}+1\right) \tilde{e}_{i_{t}}:\left(i_{t}\right)_{t \in C} \in \prod_{t \in C} B_{t}(C) \backslash \bigcup_{C^{\prime} \supset C} \prod_{t \in C} B_{t}\left(C^{\prime}\right)\right\} .
$$

In particular, for $\ell=k$ we have

$$
P_{[k]}=\left\{\left(p_{1 i_{1}}+1\right) \tilde{e}_{i_{1}}+\cdots+\left(p_{k i_{k}}+1\right) \tilde{e}_{i_{k}}:\left(i_{1}, \ldots, i_{k}\right) \in B_{1}([k]) \times \cdots \times B_{k}([k])\right\}
$$

and for $\ell=k-1$ we obtain the sets labeled by $[\hat{a}]:=\{1, \ldots, \hat{a}, \ldots, k\}$ for each $a \in[k]$ as follows

$$
\begin{aligned}
& P_{[\hat{a}]}=\left\{\left(p_{1 i_{1}}+1\right) \tilde{e}_{i_{1}}+\cdots+\left(p_{k i_{k}}+1\right) \tilde{e}_{i_{k}}:\left(i_{1}, \ldots, \widehat{i_{a}}, \ldots, i_{k}\right)\right. \\
&\left.\left.\left.\left.\quad \in B_{1}([\hat{a}]) \times \cdots \times \widehat{B_{a}([\hat{a}]}\right) \times \cdots \times B_{k}([\hat{a}]) \backslash B_{1}([k]) \times \cdots \times \widehat{B_{a}([k]}\right) \times \cdots \times B_{k}([k])\right\}\right\} .
\end{aligned}
$$

Note that when $\ell=1$, so our $d$-set contains a single element $\tilde{p}_{a}$ from $Q$, then we have by this construction that

$$
P_{\{a\}}=\left\{\left(p_{a i}+1\right) \tilde{e}_{i}: p_{a i} \geq p_{b i} \text { for all } b \neq a\right\}
$$

\section{Remarks.}

(i) Note that the sets $P_{C}$ are constructed directly in terms of the sets $B_{a}(C)$ from (10), each which is union of sets $A_{\omega}$ from (9).

(ii) The fact that $Q$ is order-generic ensures that the sets $B_{a}(C)$ are all non-empty, which in return will imply that each $P_{C}$ from (11) does not contain comparable elements, and is therefore an antichain, as we will see below.

We clearly have $S_{u}\left(D\left(Q_{*}(-\tilde{1}, \tilde{b})\right)\right) \subseteq \bigcup_{\emptyset \neq C \subseteq[k]} P_{C}$, but we have not verified that this union of these sets $P_{C}$ is actually an antichain. By the following two lemmas we will see that this is indeed the case.

Lemma 6.3. Let $Q=\left\{\tilde{p}_{1}, \ldots, \tilde{p}_{k}\right\}$ be an order-generic antichain in $\mathbb{Z}^{d}$. If $\tilde{r}=\left(p_{a_{1} i_{1}}+1\right) \tilde{e}_{i_{1}}+$ $\cdots+\left(p_{a_{\ell} i_{\ell}}+1\right) \tilde{e}_{i_{\ell}} \in P_{\left\{a_{1}, \ldots, a_{\ell}\right\}}$ and $\tilde{s}=\left(p_{b_{1} i_{1}}+1\right) \tilde{e}_{i_{1}}+\cdots+\left(p_{b_{\ell} i_{\ell}}+1\right) \tilde{e}_{i_{\ell}} \in P_{\left\{b_{1}, \ldots, b_{\ell}\right\}}$, then $\tilde{r}$ and $\tilde{s}$ are comparable in $\mathbb{Z}^{d}$ if and only if $\tilde{r}=\tilde{s}$.

Proof. Suppose $\tilde{r}<\tilde{s}$ so $\tilde{r} \neq \tilde{s}$. In this case there is a coordinate, say $i_{1}$ for simplicity, with $p_{a_{1} i_{1}}<p_{b_{1} i_{1}}$. 
Suppose that $b_{1}=a_{h} \in\left\{a_{1}, \ldots, a_{\ell}\right\}$. We first note that $h \neq 1$, since if $h=1$, then $p_{b_{1} i_{1}}=$ $p_{a_{h} i_{1}}=p_{a_{1} i_{1}}$. We then have $p_{a_{h} i_{h}}=p_{b_{1} i_{h}}>p_{b_{h} i_{h}}$ since $\tilde{s} \in P_{\left\{b_{1}, \ldots, b_{\ell}\right\}}$, and so looking at coordinates $i_{1}$ and $i_{h}$ we see that $\tilde{r}$ and $\tilde{s}$ are in this case incomparable, which is a contradiction. Therefore we have that $b_{1} \notin\left\{a_{1}, \ldots, a_{\ell}\right\}$.

By definitions of $B_{a}(C)$ in (10) we then have $i_{j} \in B_{a_{j}}\left(\left\{b_{1}, a_{1}, \ldots, a_{\ell}\right\}\right)$ for $1 \leq j \leq \ell$ and therefore $\left(i_{1}, \ldots, i_{\ell}\right) \in B_{a_{1}}\left(C^{\prime}\right) \times \cdots \times B_{a_{\ell}}\left(C^{\prime}\right)$ for $C^{\prime}=\left\{b_{1}, a_{1}, \ldots, a_{\ell}\right\} \supset\left\{a_{1}, \ldots, a_{\ell}\right\}$ contradicting $\tilde{r}=\in P_{\left\{a_{1}, \ldots, a_{\ell}\right\}}$. Hence, $\tilde{r}=\tilde{s}$ must hold. This completes the proof.

Remark. We can say a bit more than in the above proof: since $Q$ is order-generic there is an $i_{\ell+1} \in B_{b_{1}}\left(\left\{b_{1}, a_{1}, \ldots, a_{\ell}\right\}\right)$ and so

$$
\tilde{r}^{\prime}:=\tilde{r}+\left(p_{b_{1} i_{\ell+1}}+1\right) \tilde{e}_{\ell+1} \in P_{C^{\prime}}=P_{\left\{b_{1}, a_{1}, \ldots, a_{\ell}\right\}},
$$

or is dominated by an element in $P_{C^{\prime \prime}}$ where $\left\{b_{1}, a_{1}, \ldots, a_{\ell}\right\} \subseteq C^{\prime \prime}$.

The claims of Lemma 6.3 actually hold in a slightly more general setting: suppose $Q=\left\{\tilde{p}_{1}, \ldots, \tilde{p}_{k}\right\}$ is an order-generic antichain in $\mathbb{Z}^{d}$. Suppose also that $C \subseteq C^{\prime} \subseteq[k], \tilde{r} \in P_{C}, \tilde{s}^{\prime} \in P_{C^{\prime}}$ and $\tilde{r} \leq \tilde{s}^{\prime}$ in $\mathbb{Z}^{d}$. In this case we can write $C=\left\{a_{1}, \ldots, a_{\ell}\right\}$ and $C^{\prime}=\left\{b_{1}, \ldots, b_{h}\right\}$ where $\ell \leq h$. If $\tilde{r}=\left(p_{a_{1} i_{1}}+1\right) \tilde{e}_{i_{1}}+\cdots+\left(p_{a_{\ell} i_{\ell}}+1\right) \tilde{e}_{i_{\ell}} \in P_{C}$ and $\tilde{s}^{\prime}=\left(p_{b_{1} i_{1}}+1\right) \tilde{e}_{i_{1}}+\cdots+\left(p_{b_{h} i_{h}}+1\right) \tilde{e}_{i_{h}} \in P_{C^{\prime}}$, then, since $\tilde{r} \leq \tilde{s}^{\prime}$ there is a projective image $\tilde{s}$ of $\tilde{s}^{\prime}$, which we can assume has the form $\tilde{s}=$ $\left(p_{b_{1} i_{1}}+1\right) \tilde{e}_{i_{1}}+\cdots+\left(p_{b_{\ell} i_{\ell}}+1\right) \tilde{e}_{i_{\ell}}$, such that $\tilde{r} \leq \tilde{s}$. As in the proof of the previous Lemma 6.3, if $\tilde{r} \neq \tilde{s}$ then there is a coordinate, say $i_{1}$ for simplicity, with $p_{a_{1} i_{1}}<p_{b_{1} i_{1}}$. Also, since $\tilde{r}<\tilde{s}$ we cannot have $b_{1} \in\left\{a_{1}, \ldots, a_{\ell}\right\}$ and so $C^{\prime}=\left\{b_{1}, a_{1}, \ldots, a_{\ell}\right\} \subset\left\{a_{1}, \ldots, a_{\ell}\right\}$. Since $i_{j} \in B_{a_{j}}\left(C^{\prime}\right)$ for $1 \leq j \leq \ell$ we also get in this case a contradiction to the fact that $\tilde{r} \in P_{C}$. We summarize in the following Lemma.

Lemma 6.4. Let $Q=\left\{\tilde{p}_{1}, \ldots, \tilde{p}_{k}\right\}$ be an order-generic antichain in $\mathbb{Z}^{d}$ and suppose $C \subseteq C^{\prime} \subseteq[k]$, $\tilde{r} \in P_{C}$ and $\tilde{s}^{\prime} \in P_{C^{\prime}}$. If $\tilde{r} \leq \tilde{s}^{\prime}$, then $\tilde{r}=\tilde{s}$ where $\tilde{s}$ is a projective image of $\tilde{s}^{\prime}$, and this can only occur when $C^{\prime}=C$ and $\tilde{s}^{\prime}=\tilde{s}=\tilde{r}$.

By Lemmas 6.3 and 6.4 we have in particular that the union $\bigcup_{\emptyset \neq C \subseteq[k]} P_{C}$ is an antichain in $\mathbb{Z}^{d}$. We therefore have the following.

Proposition 6.5. For an order-generic antichain $Q=\left\{\tilde{p}_{1}, \ldots, \tilde{p}_{k}\right\}$ in $\mathbb{Z}^{d}$ we have

$$
S_{u}\left(D\left(Q_{*}(-\tilde{1}, \tilde{b})\right)\right)=\bigcup_{\emptyset \neq C \subseteq[k]} P_{C}
$$


where the sets $P_{C}$ are as in (11).

\section{Remarks.}

(i) Note that (14) does not hold when $Q$ is not order-generic, since the union on the right is not in general an antichain.

(ii) Although the union on the right in (14) is not, in general, disjoint, it does yield an inclusion/exclusion-like formula for explicit enumeration for an order-generic $Q$. This will be demonstrated here below for the case $k=3$.

The case when $k=3$. Here we assume that our order-generic antichain $Q=\left\{\tilde{p}_{1}, \tilde{p}_{2}, \tilde{p}_{3}\right\} \subseteq \mathbb{Z}_{0}^{d}$ contains three points. This case, as we will see, is considerably more complicated than the case $k=2$.

For distinct $i_{1}, i_{2}, i_{2} \in[d]$ and

$$
D=D\left(\tilde{p}_{1}, \tilde{p}_{2}, \tilde{p}_{3}, \tilde{b}(1), \ldots, \widehat{b}\left(\widehat{\left.i_{1}\right)}, \ldots, \widehat{\vec{b}\left(i_{2}\right)}, \ldots, \widehat{\tilde{b}\left(i_{3}\right)}, \ldots, \tilde{b}(d)\right)\right.
$$

we have by Corollary 6.2 that

$$
S_{u}(D)=\left\{\left(p_{\alpha(1) i_{1}}+1\right) \tilde{e}_{i_{1}}+\left(p_{\alpha(2) i_{2}}+1\right) \tilde{e}_{i_{2}}+\left(p_{\alpha(3) i_{3}}+1\right) \tilde{e}_{i_{3}}\right\}
$$

if there is a unique permutation $\alpha \in S_{3}$ with $p_{\alpha(1) i_{1}}<p_{\alpha(2) i_{1}}, p_{\alpha(3) i_{1}} ; p_{\alpha(2) i_{2}}<p_{\alpha(1) i_{2}}, p_{\alpha(3) i_{2}}$; and $p_{\alpha(3) i_{3}}<p_{\alpha(1) i_{3}}, p_{\alpha(2) i_{3}}$ and $S_{u}(D)=\emptyset$ otherwise.

For $\{u, v, w\}=\{1,2,3\}$ we will write the parts of $[d]$ as defined in (9) in the following way:

$$
\begin{aligned}
& A_{u, v, w}=\left\{i \in[d]: p_{u i}<p_{v i}<p_{w i}\right\} \\
& A_{u v, w}=\left\{i \in[d]: p_{u i}=p_{v i}<p_{w i}\right\} \\
& A_{u, v w}=\left\{i \in[d]: p_{u i}<p_{v i}=p_{w i}\right\}
\end{aligned}
$$

and $A_{123}=\left\{i \in[d]: p_{1 i}=p_{2 i}=p_{3 i}\right\}$. In this way our pseudo-partition of $[d]$ from in (91) becomes:

$$
\begin{gathered}
{[d]=A_{123} \cup A_{12,3} \cup A_{13,2} \cup A_{23,1} \cup A_{1,23} \cup A_{2,13} \cup A_{3,12}} \\
\cup \quad A_{1,2,3} \cup A_{1,3,2} \cup A_{2,1,3} \cup A_{2,3,1} \cup A_{3,1,2} \cup A_{3,2,1} .
\end{gathered}
$$

As $S_{u}\left(Q_{*}(-\tilde{1}, \tilde{b})\right.$ is the antichain formed by the maximal elements of all the $\left(\begin{array}{c}d+3 \\ d\end{array}\right)=\left(\begin{array}{c}d+3 \\ 3\end{array}\right)$ possible singletons $S_{u}(D)$, where $D \subseteq Q_{*}(-\tilde{1}, \tilde{b})$ contains exactly $d$ elements, then there are three types of points in $S_{u}\left(Q_{*}(-\tilde{1}, \tilde{b})\right.$ : those ones obtained from a $d$-set $D$ that contains all the three points of 
$Q=\left\{\tilde{p}_{1}, \tilde{p}_{2}, \tilde{p}_{3}\right\}$, those obtained from a $D$ that contain exactly two points of $Q$, and finally those obtained from a $D$ that contain exactly one of $\tilde{p}_{1}, \tilde{p}_{2}$ or $\tilde{p}_{3}$.

In order to facilitate our notation and presentation we define the following subsets of $[d]$, where $\{u, v, w\}=\{1,2,3\}:$

$$
\begin{aligned}
B_{\langle u, v\rangle} & :=A_{u w, v} \cup A_{w, u, v}, \\
B^{\langle u\rangle} & :=A_{v, w, u} \cup A_{w, v, u} \cup A_{v w, u}, \\
B^{\langle u *\rangle} & :=A_{w, u v} \cup A_{v, u w},
\end{aligned}
$$

that is $B_{\langle u, v\rangle}$ contains all the coordinates $i \in[d]$ where $p_{u i}<p_{v i}$ and $p_{u i}$ is not the sole minimum, $B^{\langle u\rangle}$ contains all the coordinates $i \in[d]$ where $p_{u i}$ is the sole maximum among $p_{u i}, p_{v i}$ and $p_{w i}$ and $B^{\langle u *\rangle}$ contains those coordinates $i$ where $p_{u i}$ and exactly one of the other two, $p_{v i}$ or $p_{w i}$, are the maximum ones.

By (12) the set of points in $S_{u}\left(Q_{*}(-\tilde{1}, \tilde{b})\right.$ that are formed by the sets $S_{u}(D)$ where $D \subseteq Q_{*}(-\tilde{1}, \tilde{b})$ contain all the three points of $Q=\left\{\tilde{p}_{1}, \tilde{p}_{2}, \tilde{p}_{3}\right\}$, is then given by

$$
P_{[3]}=\left\{\left(p_{1 i_{1}}+1\right) \tilde{e}_{i_{1}}+\left(p_{2 i_{2}}+1\right) \tilde{e}_{i_{2}}+\left(p_{3 i_{3}}+1\right) \tilde{e}_{i_{3}}:\left(i_{1}, i_{2}, i_{3}\right) \in B_{1}([3]) \times B_{2}([3]) \times B_{3}([3])\right\} .
$$

When $\{u, v, w\}=\{1,2,3\}$ then we have

$$
B_{u}(\{u, v\}) \times B_{v}(\{u, v\}) \backslash B_{u}([3]) \times B_{v}([3])=B_{\langle u, v\rangle} \times B_{\langle v, u\rangle} \cup B_{\langle u, v\rangle} \times B_{v}([3]) \cup B_{u}([3]) \times B_{\langle u, v\rangle}
$$

and hence the set $P_{\hat{w}}=P_{u v}$ of points in $S_{u}\left(Q_{*}(-\tilde{1}, \tilde{b})\right.$ formed by the sets $S_{u}(D)$ where $D$ contains exactly two points $\tilde{p}_{u}$ and $\tilde{p}_{v}$ are by (13) given by

$$
P_{\{u, v\}}=\left\{\left(p_{u i}+1\right) \tilde{e}_{i}+\left(p_{v j}+1\right) \tilde{e}_{j}:(i, j) \in B_{\langle u, v\rangle} \times B_{\langle v, u\rangle} \cup B_{\langle u, v\rangle} \times B_{v}([3]) \cup B_{u}([3]) \times B_{\langle u, v\rangle}\right\}
$$

Lastly, the set of points in $S_{u}\left(Q_{*}(-\tilde{1}, \tilde{b})\right.$ formed by the sets $S_{u}(D)$ where $D$ contains exactly one point $\tilde{p}_{i} \in Q$ is given by

$$
P_{\{u\}}=\left\{\left(p_{u i}+1\right) \tilde{e}_{i}: i \in B^{\langle u\rangle} \cup B^{\langle u *\rangle} \cup A_{123}\right\} .
$$

Note that for $\{u, v, w\}=\{1,2,3\}$ we have

$$
\begin{aligned}
& \left(B_{\langle u, v\rangle} \times B_{\langle v, u\rangle} \cup B_{\langle u, v\rangle} \times B_{v}([3]) \cup B_{u}([3]) \times B_{\langle u, v\rangle}\right) \\
\cap \quad & \left(B_{\langle u, w\rangle} \times B_{\langle w, u\rangle} \cup B_{\langle u, w\rangle} \times B_{v}([3]) \cup B_{u}([3]) \times B_{\langle u, w\rangle}\right) \\
= & B_{\langle u\rangle} \times A_{v w, u}
\end{aligned}
$$

and hence by Lemma 6.3 we have the following. 
Claim 6.6. Two points $\tilde{r} \in P_{\{u, v\}}$ and $\tilde{s} \in P_{\{u, w\}}$ are comparable in $\mathbb{Z}_{0}^{d}$ if and only they are equal in which case $\tilde{r}=\tilde{s}=\left(p_{u i}+1\right) \tilde{e}_{i}+\left(p_{v j}+1\right) \tilde{e}_{j}$, where $(i, j) \in B_{\langle u\rangle} \times A_{v w, u}$.

This claim together with (17) makes it possible to enumerate the maximal elements in the union $P_{\{u, v\}} \cup P_{\{u, w\}}$ in a direct manner.

By symmetry and the above Claim 6.6 we have that if two points in $P_{\{u, v\}}$ and $P_{\{v, w\}}$ respectively are comparable, then the must be equal, say to $\left(p_{u i}+1\right) \tilde{e}_{i}+\left(p_{v j}+1\right) \tilde{e}_{j}$ where $(i, j) \in A_{u w, v} \times B_{\langle v\rangle}$. Since $\left(B_{\langle u\rangle} \times A_{v w, u}\right) \cap\left(A_{u w, v} \times B_{\langle v\rangle}\right)=\emptyset$ we have, in particular, that no three points in $P_{\{u, v\}}$, $P_{\{u, w\}}$ and $P_{\{v, w\}}$ respectively, are pairwise comparable in $\mathbb{Z}_{0}^{d}$.

Lastly, consider the sets $P_{\{1\}}, P_{\{2\}}$ and $P_{\{3\}}$ from (18). Since for any distinct $u, v \in\{1,2,3\}$ we have

$$
\left(B^{\langle u\rangle} \cup B^{\langle u *\rangle} \cup A_{123}\right) \cap\left(B^{\langle v\rangle} \cup B^{\langle v *\rangle} \cup A_{123}\right)=A_{w, u v} \cup A_{123},
$$

then clearly two comparable elements in $P_{\{u\}}$ and $P_{\{v\}}$ respectively must be equal. Also note that $P_{\{1\}} \cap P_{\{2\}} \cap P_{\{3\}}=\left\{\left(p_{u i}+1\right) \tilde{e}_{i}: i \in A_{123}\right\}$.

Convention. For an upper case letter $X \in\{A, B\}$ we denote the cardinality of $X_{*}, X^{*}$ by the corresponding lower case boldface letter $\mathbf{x}_{*}$ and $\mathbf{x}^{*}$ respectively, so $\left|A_{12,3}\right|=\mathbf{a}_{12,3},\left|B^{\langle 2 *\rangle}\right|=\mathbf{b}^{\langle 2 *\rangle}$ etc.

With this convention we can now list and also enumerate the elements in $S_{u}\left(Q_{*}(-\tilde{1}, \tilde{b})\right.$ by the inclusion/exclusion principle in the following.

Proposition 6.7. For an order-generic antichain $Q=\left\{\tilde{p}_{1}, \tilde{p}_{2}, \tilde{p}_{3}\right\}$ of $\mathbb{Z}_{0}^{d}$ and the corresponding pseudo-partition of $[d]$ as in (15), we have

$$
S_{u}\left(D\left(Q_{*}(-\tilde{1}, \tilde{b})\right)\right)=P_{\{1,2,3\}} \cup P_{\{1,2\}} \cup P_{\{1,3\}} \cup P_{\{2,3\}} \cup P_{\{1\}} \cup P_{\{2\}} \cup P_{\{3\}},
$$

where $P_{\{1,2,3\}}, P_{\{u, v\}}$ and each $P_{\{u\}}$ are as in (16), (17) and (18) respectively.

Further, the cardinality is given by

$$
\begin{aligned}
& \left|S_{u}\left(D\left(Q_{*}(-\tilde{1}, \tilde{b})\right)\right)\right|=\mathbf{b}_{1}([3]) \mathbf{b}_{2}([3]) \mathbf{b}_{3}([3])+\mathbf{b}_{\langle 1,2\rangle} \mathbf{b}_{\langle 2,1\rangle}+\mathbf{b}_{\langle 1,2\rangle} \mathbf{b}_{\langle 2\rangle}+\mathbf{b}_{\langle 1\rangle} \mathbf{b}_{\langle 2,1\rangle} \\
& \quad+\mathbf{b}_{\langle 1,3\rangle} \mathbf{b}_{\langle 3,1\rangle}+\mathbf{b}_{\langle 1,3\rangle} \mathbf{b}_{\langle 3\rangle}+\mathbf{b}_{\langle 1\rangle} \mathbf{b}_{\langle 3,1\rangle}+\mathbf{b}_{\langle 2,3\rangle} \mathbf{b}_{\langle 3,2\rangle}+\mathbf{b}_{\langle 2,3\rangle} \mathbf{b}_{\langle 3\rangle}+\mathbf{b}_{\langle 2\rangle} \mathbf{b}_{\langle 3,2\rangle} \\
& \quad-\quad\left(\mathbf{b}_{\langle 1\rangle} \mathbf{a}_{23,1}+\mathbf{b}_{\langle 2\rangle} \mathbf{a}_{13,2}+\mathbf{b}_{\langle 3\rangle} \mathbf{a}_{12,3}\right)+\mathbf{b}^{\langle 1\rangle}+\mathbf{b}^{\langle 2\rangle}+\mathbf{b}^{\langle 3\rangle}+\mathbf{a}_{3,12}+\mathbf{a}_{2,13}+\mathbf{a}_{1,23}+\mathbf{a}_{123}
\end{aligned}
$$

Remark. Unlike the presentation in Proposition 5.8 and Corollary 5.9 the presentation for the antichain $S_{u}\left(D\left(Q_{*}(-\tilde{1}, \tilde{b})\right)\right)$ as a union in Proposition 6.7 is symmetric, however it is not a disjoint union, as that would be quite convoluted and confusing. 
Corollary 6.8. For an order-generic antichain $Q=\left\{\tilde{p}_{1}, \tilde{p}_{2}, \tilde{p}_{3}\right\}$ of $\mathbb{Z}_{0}^{d}$, the unique local zero dimensional type 3 monomial ideal $I$ of $R=K\left[x_{1}, \ldots, x_{d}\right]$ with $\operatorname{\partial oc}(I)=\left\{\tilde{x}^{\tilde{p}_{1}}, \tilde{x}^{\tilde{p}_{2}}, \tilde{x}^{\tilde{p}_{3}}\right\}$ is given by

$$
I=\left(\tilde{x}^{\tilde{r}}: \tilde{r} \in S_{u}\left(D\left(Q_{*}(-\tilde{1}, \tilde{b})\right)\right)\right\}
$$

where $S_{u}\left(D\left(Q_{*}(-\tilde{1}, \tilde{b})\right)\right)$ is as in Proposition 6.7.

\section{Remarks.}

(i) Corollary 6.8 characterizes completely those monomial ideals $I$ of $R=K\left[x_{1}, \ldots, x_{d}\right]$ that make $R / I$ a local Artinian ring of type 3, where $\partial \mathrm{oc}(I)$ corresponds to an order-generic antichain in $\mathbb{Z}^{d}$ in a purely combinatorial way, using only the poset structure of $\mathbb{Z}^{d}$.

(ii) Note that since $S_{u}\left(D\left(Q_{*}(-\tilde{1}, \tilde{b})\right)\right)$ is an antichain, then $I$ as presented in Corollary 6.8 is exactly its minimal generation, that is, $\left\{\tilde{x}^{\tilde{r}}: \tilde{r} \in S_{u}\left(D\left(Q_{*}(-\tilde{1}, \tilde{b})\right)\right)\right\}$ is the unique Gröbner basis for $I$.

\section{Socles of GENERAL IDEALS}

We have so far only been interested in the combinatorial properties of monomial ideals of $R=$ $K\left[x_{1}, \ldots, x_{d}\right]$ that can described solely in terms of the monoid $\left[x_{1}, \ldots, x_{d}\right]$ viewed as a poset, where the partial order is given by divisibility. As a consequence, the role of the field $K$ has so far not played a major role in our investigations on monomial ideals. We have at times further assumed our monomial ideal $I \subseteq R$ to be positive, that is $I \subseteq\left(x_{1} \cdots x_{d}\right)$, or each (generating) monomial $\tilde{x}^{\tilde{p}}$ of $I$ having $\tilde{p} \in \mathbb{N}^{d}$. Also, we so far only considered the the socle $\operatorname{Soc}(I)=\operatorname{Soc}_{\mathbf{m}}(I)$ of $R$ w.r.t. the specific maximal ideal $\mathbf{m}=\left(x_{1}, \ldots, x_{d}\right) \subseteq R$.

Just to quickly iterate that we are not gaining anything by allowing $K$-linear combination when considering monomial ideals, we note that that for a monomial ideal $I=\left(\tilde{x}^{\tilde{p}_{1}}, \ldots, \tilde{x}^{\tilde{p}_{k}}\right) \subseteq R$ the set of its generators $M=\left\{\tilde{x}^{\tilde{p}_{1}}, \ldots, \tilde{x}^{\tilde{p}_{k}}\right\}$ is always a Gröbner basis for $I$ w.r.t. any term order on $\left[x_{1}, \ldots, x_{d}\right]$. As a result, a fully reduced polynomial $f \in R \backslash\{0\}$ is exactly a linear combination of fully reduced monomials w.r.t. $I$, that is, those monomials that do not reduce to zero in $R / I$. In particular we have the following.

Observation 7.1. If $I \subseteq R=K\left[x_{1}, \ldots, x_{d}\right]$ is a monomial ideal, then the socle of $R / I$ w.r.t. the maximal ideal $\mathbf{m}=\left(x_{1}, \ldots, x_{d}\right)$ is exactly the set of all $K$-linear combinations of the monomials of the socle, $\operatorname{Soc}(I)=\operatorname{Span}_{K}\left(\left\{\tilde{x}^{\tilde{r}}: \tilde{x}^{\tilde{r}} \in \operatorname{Soc}(I)\right\}\right)$. 
In this section we show that even in these mentioned seemingly restrictive case, we have managed to capture many of the interesting combinatorial properties of the socle of a general ideal w.r.t. a maximal ideal.

First we recall some notations and facts from commutative ring theory. For an ideal $I \subseteq R$ let $\sqrt{I}=\left\{f \in R: f^{n} \in I\right.$ for some $\left.n \in \mathbb{N}\right\}$ denote the radical of $I$. For a field $K$ and $R=K\left[x_{1}, \ldots, x_{d}\right]$ then $V(I)=\left\{\tilde{a} \in K^{d}: f(\tilde{a})=0\right.$ for all $\left.f \in I\right\}$ is the affine variety defined by $I$. For a subset $U \subseteq K^{d}$ we let $I(U)=\{f \in R: f(\tilde{a})=0$ for all $\tilde{a} \in U\}$ denote the ideal of all polynomials in $R$ that vanish on $U$. For any field $K$ and a point $\tilde{a} \in K^{d}$ then clearly $\left(x_{1}-a_{1}, \ldots, x_{d}-a_{d}\right) \subseteq R$ is a maximal ideal. For the converse, we recall an important corollary of Hilbert's Nullstellensatz [AM69, p. 85] and Alu09, p. 410] as the following [Alu09, Cor. 2.10, p. 406].

Theorem 7.2. If $K$ is an algebraically closed field, then an ideal of $K\left[x_{1}, \ldots, x_{d}\right]$ is maximal if and only if it has the form $\mathbf{m}_{\tilde{a}}=\left(x_{1}-a_{1}, \ldots, x_{d}-a_{d}\right)$ for some $\tilde{a} \in K^{d}$.

Recall the following theorem from [AM69, Prop. 1.11] on prime avoidance:

Theorem 7.3. Let $\mathbf{p}_{1}, \ldots, \mathbf{p}_{k}$ be prime ideals in a commutative ring. If $I \subseteq \bigcup_{i=1}^{k} \mathbf{p}_{i}$ is an ideal, then $I \subseteq \mathbf{p}_{i}$ for some $i$.

Since $R=K\left[x_{1}, \ldots, x_{d}\right]$ is Noetherian, every ideal $I \subseteq R$ has a primary decomposition $I=$ $\bigcap_{i=1}^{k} \mathbf{q}_{i}$ which we can assume to be minimal (or reduced). For each $i$ let $\mathbf{p}_{i}=\sqrt{\mathbf{q}_{i}}$ be the associated prime ideal. The set $\left\{\mathbf{p}_{1}, \ldots, \mathbf{p}_{k}\right\}$ of associated primes of the ideal $I$ is uniquely determined by $I$ and is always a finite set.

Suppose $\mathbf{m} \subseteq R$ is a maximal ideal containing the ideal $I$ is $\operatorname{such}$ that $\operatorname{Soc}_{\mathbf{m}}(I)$ is nontrivial, so $\operatorname{Soc}_{\mathbf{m}}(I)=(I: \mathbf{m}) \neq I$. Since

$$
I \neq(I: \mathbf{m})=\bigcap_{f \in \mathbf{m}}(I: f)
$$

then for each $f \in \mathbf{m}$ we have $(I: f) \neq I$ as well, and so by [AM69, Prop. 4.7] we then have

$$
\mathbf{m} \subseteq\{f \in R:(I: f) \neq I\}=\bigcup_{i=1}^{k} \mathbf{p}_{i} .
$$

By Theorem 7.3 above we therefore have that $\mathbf{m} \subseteq \mathbf{p}_{i}$ for some $i$, and since $\mathbf{m}$ is maximal we have $\mathbf{m}=\mathbf{p}_{i}$. We summarize in the following.

Proposition 7.4. If $R$ is a Noetherian ring, $I \subseteq R$ an ideal and $\mathbf{m} \subseteq R$ a maximal ideal containing I such that $\operatorname{Soc}_{\mathbf{m}}(I)=(I: \mathbf{m}) \neq I$, then $\mathbf{m}$ is one of the finitely many associated prime ideals of $I$. 
If now $I \subseteq R=K\left[x_{1}, \ldots, x_{d}\right]$ is a monomial ideal then every associated prime of $I$ is a face ideal, or a Stanley-Reisner ideal [MS05, p. 19], that is an ideal of the form $\left(x_{i_{1}}, \ldots, x_{i_{h}}\right)$ (See [Vil01, Prop. 5.1.3].) Hence, if $\mathbf{m} \subseteq R$ is a maximal ideal such that $\operatorname{Soc}_{\mathbf{m}}(I) \neq I$, then by Proposition 7.4 $\mathbf{m}$ must be a face ideal and so we have the following.

Proposition 7.5. If $K$ is a field, $I \subseteq R=K\left[x_{1}, \ldots, x_{d}\right]$ a monomial ideal and $\mathbf{m} \subseteq R$ a maximal ideal containing $I$ such that $\operatorname{Soc}_{\mathbf{m}}(I)=(I: \mathbf{m}) \neq I$, then $\mathbf{m}=\left(x_{1}, \ldots, x_{d}\right)$.

If now $K$ is algebraically closed, then by Theorem 7.2 we have a bijective correspondence between points $\tilde{a} \in K^{d}$ and maximal ideals $\mathbf{m}_{\tilde{a}}=\left(x_{1}-a_{1}, \ldots, x_{d}-a_{d}\right)$ of $R=K\left[x_{1}, \ldots, x_{d}\right]$.

Observation 7.6. Let $R=K\left[x_{1}, \ldots, x_{d}\right]$ where $K$ is algebraically closed and $I \subseteq R$ a proper ideal. A maximal ideal $\mathbf{m}$ of $R$ contains $I$ if and only if $\mathbf{m}=\mathbf{m}_{\tilde{a}}$ for some $\tilde{a} \in V(I)$.

For an ideal $I \subseteq R=K\left[x_{1}, \ldots, x_{d}\right]$ where $K$ is algebraically closed and $\mathbf{m}_{\tilde{a}}$ a maximal ideal containing $I$ let $\operatorname{Soc}_{\tilde{a}}(I):=\operatorname{Soc}_{\mathbf{m}_{\tilde{a}}}(I)=\left(I: \mathbf{m}_{\tilde{a}}\right)$ denote the socle of $I$ w.r.t. the maximal ideal $\mathbf{m}_{\tilde{a}}$. By Proposition 7.4 the following.

Corollary 7.7. If $K$ is an algebraically closed field and $I \subseteq R=K\left[x_{1}, \ldots, x_{d}\right]$ is an ideal of $R$, then for all but finitely many points $\tilde{a} \in V(I)$ the socle $\operatorname{Soc}_{\tilde{a}}(I)=I$ is trivial.

Also, directly by Proposition 7.5 do we have the following corollary.

Corollary 7.8. If $K$ is an algebraically closed field, $I \subseteq R=K\left[x_{1}, \ldots, x_{d}\right]$ is a monomial ideal of $R$ and $\operatorname{Soc}_{\tilde{a}}(I) \neq I$, then $\tilde{a}=\tilde{0}$.

As the combinatorial properties we want to investigate can be phrased in terms of the monoid $\left[x_{1}, \ldots, x_{d}\right]$ of indeterminates as a poset, we obtain virtually the same combinatorial properties of our monomial ideal $I \subseteq R=K\left[x_{1}, \ldots, x_{d}\right]$ regardless of what the field $K$ is. Hence, we might as well look at the extended ideal $\bar{I}=I \bar{K}\left[x_{1}, \ldots, x_{d}\right]$ where $\bar{K}$ is the algebraic closure of $K$. This is so, since for any vector space (in particular ideals) $W$ over $K$, then $B$ is a basis for $W$ as a vector space over $K$ if, and only if, $B$ is a basis for $W \otimes_{K} \bar{K}$ as a vector space over $\bar{K}$. In particular $\bar{R}:=\bar{K}\left[x_{1}, \ldots, x_{d}\right]=\bar{K} \otimes_{K} K\left[x_{1}, \ldots, x_{d}\right]$, and in general for any ideal $I \subseteq R$ the corresponding ideal $\bar{I}$ of $\bar{R}$ has the form $\bar{I}=I \otimes_{K} \bar{K}$. Hence, as we have been almost exclusively focusing on the bases of the socle of a monomial ideal consisting of monomials alone, we will not lose any of the combinatorial poset structure of the bases by tensoring with $\bar{K}$, that is, assuming $K$ is algebraically 
closed. In that case, as stated earlier, Theorem 7.2 yields a bijective correspondence between points of $K^{d}$ and maximal ideals of $R$.

At this point two natural questions arise:

(1) What ideals $I \subseteq R$ have their socles $\operatorname{Soc}_{\tilde{a}}(I)$ trivial for all $\tilde{a} \in V(I)$ ?

(2) Knowing just the socle $\operatorname{Soc}_{\tilde{a}}(I)$ of an a priori unknown ideal $I$ of $R$, can we retrieve the ideal $I$ ?

In general, the second question has a negative answer. However, in numerous specific cases the answer is positive, for example if we know $I$ has dimension zero, or if we know the socle to be trivial.

We consider the first question for $I=(f)$ a principal ideal of $R=K\left[x_{1}, \ldots, x_{d}\right]$. Since $R$ is a UFD we can write $f=r_{1}^{b_{1}} \cdots r_{h}^{b_{h}}$ where each $r_{i}$ is irreducible in $R$. In this case we have the following minimal primary decomposition of $I$ as

$$
I=\bigcap_{i=1}^{h}\left(r_{i}^{b_{i}}\right),
$$

and the unique set of associated primes $\left\{\mathbf{p}_{1}, \ldots, \mathbf{p}_{h}\right\}$ where $\mathbf{p}_{i}=\sqrt{\left(r_{i}^{b_{i}}\right)}=\left(r_{i}\right)$ for each $i$. For $d \geq 2$ then clearly none of these principal prime ideals $\mathbf{p}_{i}$ are maximal ideals of $R$, and hence, by Proposition 7.4, $\operatorname{Soc}_{\tilde{a}}(I)$ must be trivial.

Observation 7.9. If $I \subseteq R=K\left[x_{1}, \ldots, x_{d}\right]$ is a principal ideal where $K$ is algebraically closed and $d \geq 2$, then $\operatorname{Soc}_{\tilde{a}}(I)=I$ is trivial for all $\tilde{a} \in V(I)$.

When $I$ is generated by two or more elements from $R$, things are, needless to say, more involved, and it seems we must apply the Euclidean algorithm to obtain Gröbner bases for the ideal, in order to conclude something fruitful - we leave that as a question in the next section.

\section{Summary AND FURTher QUestions}

We briefly discuss some of the main results in this article and post some some relevant and motivating questions.

Presenting everything in terms of $\mathbb{Z}^{d}$ or $\mathbb{N}_{0}^{d}$ provided with the componentwise partial order, we showed in Theorem 3.2 and its slight generalization Theorem 3.4 that a given antichain $G$ of $\mathbb{Z}^{d}$ (corresponding to a monomial ideal of the polynomial ring in $d$ variables) can always be retrieved from the set $S_{d}\left(U\left(G^{*}\right)\right)$ alone, or more generally from $S_{d}\left(U\left(G^{*}(\tilde{a} . \tilde{b})\right)\right)$ as stated in Theorem 3.4 . 
As a consequence, the monomial ideal $I$ can always be retrieved from a specific derived version of $\partial \operatorname{oc}(I)$ and also from a more general derived version of it socle as stated in Theorem 3.4.

The main results in Section 4 are Theorem 4.2 and Theorem 4.3 which are duals of the previously mentioned Theorems 3.2 and 3.4 . This shows that any antichain $Q$ in $\mathbb{N}^{d}$ is indeed the socle for a monomial ideal whose minimal generators correspond to $U\left(S_{u}\left(D\left(Q_{*}\right)\right)\right)$ or more generally to $U\left(S_{u}\left(D\left(Q_{*}(\tilde{a}, \tilde{b})\right)\right)\right)$ in Theorem [3.4. As a consequence, for any set of incomparable monomials in the monoid $\left[x_{1}, \ldots, x_{d}\right]$ w.r.t. divisibility, there are monomials ideals $I$ with the given set of monomials as $\partial \mathrm{oc}(I)$.

In Section 5 we focused on Artinian monomial ideals and obtained some structure theorems for zero dimensional local type $k \operatorname{rings} R / I$ where $I$ is a monomial ideal and $k=1,2$.

In Section 6] we obtained some results for zero dimensional local type $k$ rings $R / I$ when $k \geq 3$ and where we assumeed $\partial \mathrm{oc}(I)$, or rather the power points of the corresponding monomials, to be order-generic. This yielded a specific enumeration of the minimal generators of $I$ in Proposition 6.7 .

In the last Section 7 we stated some observations about socles of general ideals of Noetherian rings and argued that a lot of the interesting combinatorics really is captured by monomial ideals.

There are still many questions worth considering related to what has been covered.

Question 8.1. Let $K$ be algebraically closed, and $I \subseteq R=K\left[x_{1}, \ldots, x_{d}\right]$ an ideal such that that $\operatorname{Soc}_{\tilde{a}}(I)=I$ is trivial for all $\tilde{a} \in V(I)$. Can we conclude something about the Gröbner basis for $I$ w.r.t. some term order?

Question 8.2. Can we generalize the results to general ideals, using Gröbner bases in terms of some term orders? If not, perhaps one can obtain similar results for toric ideals, since there all relations can be obtained and described within the monoid $\left[x_{1}, \ldots, x_{d}\right] \subseteq R$.

Question 8.3. When translating the results in previous sections into results for polynomial rings $R=K\left[x_{1}, \ldots, x_{d}\right]$, or general Noetherian rings for that matter, and their ideals, can anything be phrased simpler by using the ring structure instead or in addition to the partial order on the monomials induced by divisibility?

\section{REFERENCES}

\section{[A00] https://oeis.org/A000670}

[AF74] Frank W. Anderson and Kent R. Fuller, Rings and categories of modules, Graduate Texts in Mathematics, vol. 13, Springer-Verlag, New York-Heidelberg, 1974. 
[Agn97] Geir Agnarsson, The number of outside corners of monomial ideals, J. Pure Appl. Algebra 117/118 (1997), 3-21.

[Agn00] _ Co-generators for algebras over fields and commutative applications, Comm. Algebra 28 (2000), no. 9, 4071-4087.

[AL94] William W. Adams and Philippe Loustaunau, An introduction to Gröbner bases, Graduate Studies in Mathematics, vol. 3, Amer. Math. Soc., Providence, RI, 1994.

[Alu09] Paolo Aluffi, Algebra: chapter 0, Graduate Studies in Mathematics, vol. 104, Amer. Math. Soc., Providence, RI, 2009.

[AM69] Michael Francis Atiyah and Ian G. Macdonald, Introduction to commutative algebra, Addison-Wesley, Reading, Mass.-London-Don Mills, Ont., 1969.

[Bei93] Mark B. Beintema, A note on Artinian Gorenstein algebras defined by monomials, Rocky Mountain J. Math. 23 (1993), no. 1, 1-3.

[BH97] Winfried Bruns and Jürgen Herzog, Cohen-Macaulay rings, revised ed., Cambridge Studies in Advanced Mathematics, no. 39, Cambridge Univ. Press, Cambridge, 1997.

[BPS98] Dave Bayer, Irena Peeva, and Bernd Sturmfels, Monomial resolutions, Math. Res. Lett. 5 (1998), no. 1-2, 31-46.

[BW93] Thomas Becker and Volker Weispfenning, Gröbner bases, Graduate Texts in Mathematics, vol. 141, SpringerVerlag, New York, 1993, A computational approach to commutative algebra. In cooperation with Heinz Kredel.

[Eis95] David Eisenbud, Commutative algebra with a view toward algebraic geometry, Graduate Texts in Mathematics, vol. 150, Springer-Verlag, New York, 1995.

[GP06] Edgar G. Goodaire and Michael M. Parmenter, Discrete mathematics with graph theory, 3rd ed., Pearson Prentice Hall, 2006.

[HV06] Craig Huneke and Adela Vraciu, Rings that are almost Gorenstein, Pacific J. Math. 225 (2006), no. 1, $85-102$.

[Mac94] Francis Sowerby Macaulay, The algebraic theory of modular systems, Cambridge Mathematical Library, Cambridge Univ. Press, Cambridge, 1994, Revised reprint of the 1916 original. With an introduction by Paul Roberts.

[MS05] Ezra Miller and Bernd Sturmfels, Combinatorial commutative algebra, Graudate Texts in Mathematics, vol. 227, Springer-Verlag, New York, 2005.

[OBn] https://en.wikipedia.org/wiki/Ordered_Bell_number.

[Sca86] Herbert E. Scarf, Neighborhood systems for production sets with indivisibilities, Econometrica 54 (1986), no. $3,507-534$.

[Sti] https://en.wikipedia.org/wiki/Stirling_numbers_of_the_second_kind

[Vil01] Rafael H. Villarreal, Monomial algebras, Monographs and Textbooks in Pure and Applied Mathematics, vol. 238, Marcel Dekker, Inc., New York, 2001.

[Wol16] Anna-Rose G. Wolff, The survival complex, arXiv:1602.08998 [math.AC], 2016. 
Department of Mathematical Sciences, George Mason University, Fairfax, VA 22030

E-mail address: geir@math.gmu.edu

Department of Mathematical Sciences, George Mason University, Fairfax, VA 22030

E-mail address: nepstei2@gmu.edu 